\title{
The Addition of a Cyclopropyl Alkyne to an Asymmetrically- Substituted Disilene: A Mechanistic Study
}

Andrew T. Henry, ${ }^{\dagger}$ Isabell Vacirca, ${ }^{\ddagger}$ Jeremy L. Bourque, ${ }^{\dagger}$ David Scheschkewitz, ${ }^{\ddagger}$ Kim. M. Baines $^{\dagger *}$

†Department of Chemistry, University of Western Ontario, London, Ontario, Canada N6A 5B7

†Krupp-Chair of General and Inorganic Chemistry, Saarland University, 66125 Saarbrücken, Germany.

\section{Supporting Information}

\section{Table of Contents}

Table S1: Comparison of ${ }^{1} \mathrm{H},{ }^{13} \mathrm{C}$, and ${ }^{29} \mathrm{Si}$ chemical shifts for $7,8,11,12 \ldots \ldots \ldots \ldots \ldots \ldots \ldots . . . . . . . . . . . .33$

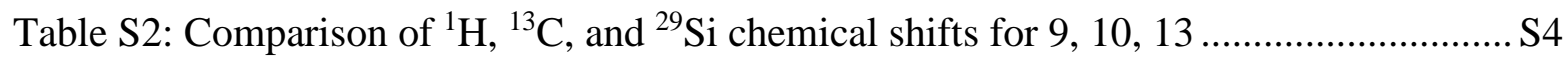

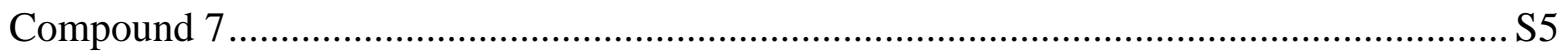

Figure S1: a) ${ }^{1} \mathrm{H}$ NMR spectrum (600 MHz, $\mathrm{C}_{6} \mathrm{D}_{6}$ ) of 7; b) Expansion from 6.70 - 7.12 ppm;

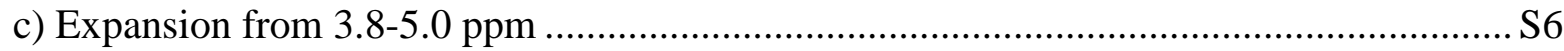

Figure S3: Expansion of the ${ }^{13} \mathrm{C}-{ }^{1} \mathrm{H}$ gHSQCAD NMR spectrum of 7 (600 MHz, $\mathrm{C}_{6} \mathrm{D}_{6}$ ) with ${ }^{1} \mathrm{H}$; ${ }^{13} \mathrm{C}$ ranges of: (0.0 - $1.8 \mathrm{ppm} ; 20$ - $\left.31 \mathrm{ppm}\right)(2.0$ - 5.0 ppm; 30 - 60 ppm) (4.0 - 4.8 ppm; 90 - 125 ppm) (6.6 - 8.2 ppm; $120-145$ ppm).

Figure S4: Expansion of the ${ }^{13} \mathrm{C}-{ }^{1} \mathrm{H}$ gHMBC NMR spectrum of 7 (600 $\mathrm{MHz}, \mathrm{C}_{6} \mathrm{D}_{6}$ ) with ${ }^{1} \mathrm{H} ;{ }^{13} \mathrm{C}$ ranges of: $(0.0-1.7 \mathrm{ppm} ; 148-157 \mathrm{ppm})(4.45-4.80 \mathrm{ppm} ; 144-147 \mathrm{ppm})(6.55-$ 7.35 ppm; 119 - 146 ppm) (8.12 - 8.21 ppm; 125 - 140 ppm)....

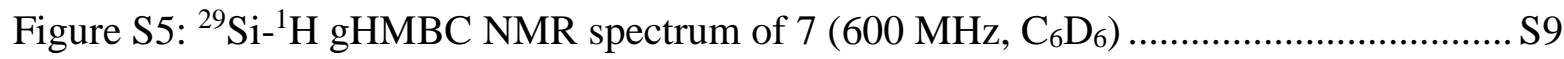

Compound 8 . S10

Figure S6: a) ${ }^{1} \mathrm{H}$ NMR spectrum of 8 (600 MHz, $\mathrm{C}_{6} \mathrm{D}_{6}$ ); b) Expansion from 6.69 - 6.71 ppm; c) Expansion from 3.6 - 4.1 ppm; d) Expansion from $1.30-1.35$ ppm

Figure S7: Expansion of the ${ }^{1} \mathrm{H}^{-1} \mathrm{H}$ COSY NMR spectrum of 8 (600 MHz, ${ }_{6} \mathrm{D}_{6}$ ) illustrating correlations among the Si-Ph hydrogens (f1: $6.5-8.5 \mathrm{ppm}$; f2: $6.6-8.3 \mathrm{ppm}$ ), the C-Ph hydrogens (f1: 6.0 - $7.4 \mathrm{ppm}$; f2: 6.1 - $7.7 \mathrm{ppm}$ ), and the correlation between the $\mathrm{CH}-\mathrm{OMe}$ and $\mathrm{CH}-\mathrm{Ph}$ hydrogens $(\mathrm{f} 1=\mathrm{f} 2: 3.4-4.3 \mathrm{ppm})$,. S12 
Figure S8: Expansion of the ${ }^{13} \mathrm{C}-{ }^{1} \mathrm{H}$ gHSQCAD NMR spectrum of $8\left(600 \mathrm{MHz}, \mathrm{C}_{6} \mathrm{D}_{6}\right)$ with ${ }^{1} \mathrm{H} ;{ }^{13} \mathrm{C}$ ranges of $(0.0-1.9 \mathrm{ppm} ; 17-32 \mathrm{ppm})(2.5-4.9 \mathrm{ppm} ; 30-55 \mathrm{ppm})(6.0-8.3 \mathrm{ppm}$; 120 - $141 \mathrm{ppm})(3.7-6.8 \mathrm{ppm} ; 91-93 \mathrm{ppm})$. S13

Figure S9: Expansions of the ${ }^{13} \mathrm{C}-{ }^{1} \mathrm{H}$ gHMBC NMR spectrum of $8\left(600 \mathrm{MHz}, \mathrm{C}_{6} \mathrm{D}_{6}\right) \ldots \ldots . \mathrm{S} 14$

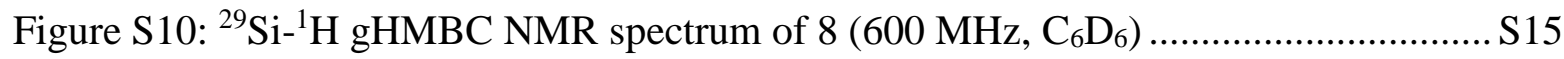

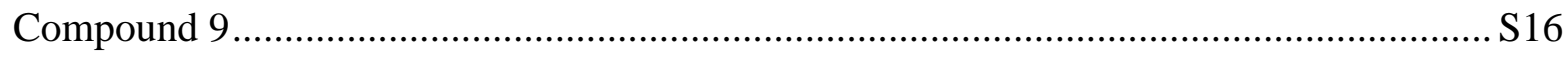

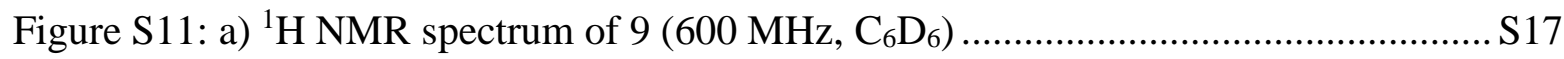

Figure S12: Expansion of the ${ }^{13} \mathrm{C}-{ }^{1} \mathrm{H}$ gHSQC NMR spectrum of $9\left(600 \mathrm{MHz}, \mathrm{C}_{6} \mathrm{D}_{6}\right) \ldots . . . \mathrm{S} 18$

Figure S13: Expansion of the ${ }^{13} \mathrm{C}-{ }^{1} \mathrm{H}$ gHMBC NMR spectrum of $9\left(600 \mathrm{MHz}, \mathrm{C}_{6} \mathrm{D}_{6}\right) \ldots \ldots . \mathrm{S} 19$

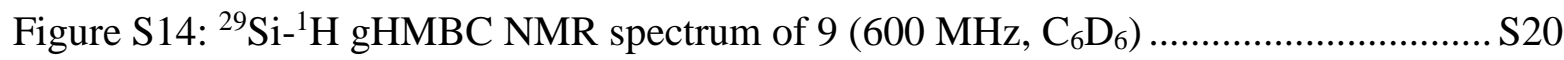

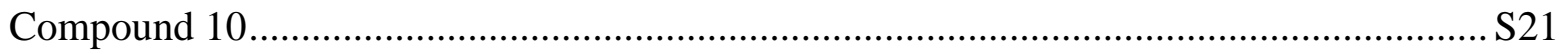

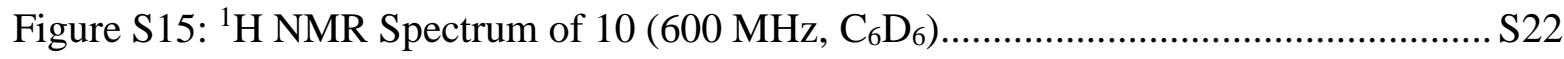

Figure S16: Expansions of the ${ }^{13} \mathrm{C}-{ }^{1} \mathrm{H}$ gHSQCAD NMR spectrum of $10\left(600 \mathrm{MHz}, \mathrm{C}_{6} \mathrm{D}_{6}\right)$

Figure S17: Expansions of the ${ }^{13} \mathrm{C}-{ }^{1} \mathrm{H}$ gHMBC NMR spectrum of $10\left(600 \mathrm{MHz}, \mathrm{C}_{6} \mathrm{D}_{6}\right)$.. S24

Figure S18: ${ }^{29} \mathrm{Si}-{ }^{1} \mathrm{H}$ gHMBC NMR spectrum of $10\left(600 \mathrm{MHz}, \mathrm{C}_{6} \mathrm{D}_{6}\right)$................................ S25

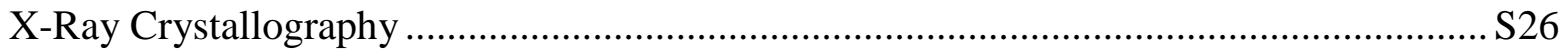

Table S3: Summary of crystallographic parameters for 7 ........................................ S27

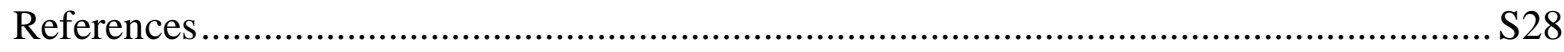


Table S1: Comparison of ${ }^{1} \mathrm{H},{ }^{13} \mathrm{C}$, and ${ }^{29} \mathrm{Si}$ chemical shifts for 7, 8, 11, 12

\begin{tabular}{|c|c|c|c|c|}
\hline & $\underbrace{\text { Mes }}_{\mathbf{1 1}^{\text {a }}}$ & $\underbrace{\text { Mes, Mes }}_{\mathbf{1 2}^{\text {a }}}$ & $\underbrace{\text { Tip }}_{7}$ & $\underbrace{\mathrm{Tip}}_{\mathbf{O M e}}$ \\
\hline Solvent & $\mathrm{C}_{6} \mathrm{D}_{6}$ & $\mathrm{C}_{6} \mathrm{D}_{6}$ & $\mathrm{C}_{6} \mathrm{D}_{6}$ & $\mathrm{C}_{6} \mathrm{D}_{6}$ \\
\hline \multicolumn{5}{|c|}{${ }^{1}$ H NMR Chemical Shifts } \\
\hline$\underline{\mathbf{H} C P h}$ & $\begin{array}{l}4.81 \\
{[\mathrm{~d}, \mathrm{~J}=12 \mathrm{~Hz}]}\end{array}$ & $\begin{array}{l}4.49 \\
{[\mathrm{~d}, \mathrm{~J}=10 \mathrm{~Hz}]}\end{array}$ & $\begin{array}{l}4.93 \\
{[\mathrm{~d}, \mathrm{~J}=10 \mathrm{~Hz}]}\end{array}$ & 4.02 \\
\hline$\underline{\mathbf{H}} \mathrm{COCH}_{3}$ & $\begin{array}{l}4.81 \\
{[\mathrm{~d}, \mathrm{~J}=12 \mathrm{~Hz}]}\end{array}$ & $\begin{array}{l}4.17 \\
{[\mathrm{~d}, \mathrm{~J}=10 \mathrm{~Hz}]}\end{array}$ & $\begin{array}{l}4.08 \\
{[\mathrm{~d}, \mathrm{~J}=10 \mathrm{~Hz}]}\end{array}$ & 3.71 \\
\hline $\mathrm{CH}_{3}$ & 1.94 & 1.32 & - & 1.33 \\
\hline $\mathrm{OC}_{3}$ & 2.89 & 2.93 & 2.20 & 3.02 \\
\hline $\mathrm{C}=\overline{\mathrm{C}}=\mathrm{C} \underline{\mathbf{H}}$ & 5.72 & 6.22 & - & 6.71 \\
\hline \multicolumn{5}{|c|}{${ }^{13}$ C NMR Chemical Shifts } \\
\hline $\mathrm{H} \underline{\mathrm{CP}} \mathrm{Ph}$ & $52.42^{b}$ & 44.1 & 42.30 & 54.1 \\
\hline $\mathrm{H} \underline{\mathbf{C}} \mathrm{OCH}_{3}$ & $77.07^{\mathrm{b}}$ & 84.4 & 91.24 & 91.7 \\
\hline $\mathrm{O} \underline{\mathbf{C}} \mathrm{H}_{3}$ & $56.62^{\mathrm{b}}$ & 51.9 & 57.42 & 56.8 \\
\hline $\mathrm{C}=\underline{\mathrm{C}}=\mathrm{C}$ & $213.14^{\mathrm{b}}$ & 210.2 & - & 218.4 \\
\hline \multicolumn{5}{|c|}{${ }^{29}$ Si NMR Chemical Shifts } \\
\hline $\mathrm{PhC}(\mathrm{H}) \underline{\mathbf{S}}$ & -25.2 & -18.1 & -12 & -28 \\
\hline $\mathrm{C}=\mathrm{C}(\mathrm{H}) \underline{\mathbf{S}} \underline{\mathbf{i}}$ & -10.0 & -13.4 & -32 & -31 \\
\hline
\end{tabular}

${ }^{a}$ from reference $1{ }^{\mathrm{b}}$ in $\left(\mathrm{CD}_{3}\right)_{2} \mathrm{C}=\mathrm{O}$ 
Table S2: Comparison of ${ }^{1} \mathrm{H},{ }^{13} \mathrm{C}$, and ${ }^{29} \mathrm{Si}$ chemical shifts for $\mathbf{9}, \mathbf{1 0}, \mathbf{1 3}$

\begin{tabular}{|c|c|c|c|}
\hline & Mes Mes & (Tip & (10) \\
\hline Solvent & $\mathrm{C}_{6} \mathrm{D}_{6}$ & $\mathrm{C}_{6} \mathrm{D}_{6}$ & $\mathrm{C}_{6} \mathrm{D}_{6}$ \\
\hline \multicolumn{4}{|c|}{${ }^{1} \mathrm{H}$ NMR Chemical Shifts } \\
\hline$\underline{\mathbf{H C P h}}$ & 2.31 & 2.84 & 2.66 \\
\hline & {$[\mathrm{d}, \mathrm{J}=5 \mathrm{~Hz}]$} & {$[\mathrm{d}, \mathrm{J}=4.7 \mathrm{~Hz}]$} & {$[\mathrm{d}, \mathrm{J}=5.8 \mathrm{~Hz}]$} \\
\hline$\underline{\mathbf{H}} \mathrm{COCH}_{3}$ & 3.98 & 3.60 & 3.8 \\
\hline & {$[\mathrm{d}, \mathrm{J}=5 \mathrm{~Hz}]$} & {$[\mathrm{d}, \mathrm{J}=4.7 \mathrm{~Hz}]$} & \\
\hline $\mathrm{C}_{3}$ & 1.60 & 1.25 & 1.59 \\
\hline $\mathrm{OC} \underline{\mathbf{H}}_{3}$ & 3.17 & 3.13 & 3.26 \\
\hline $\mathrm{C}=\mathrm{C} \underline{\mathbf{H}}$ & 6.88 & 7.46 & 7.00 \\
\hline \multicolumn{4}{|c|}{${ }^{13}$ C NMR Chemical Shifts } \\
\hline $\mathrm{H} \underline{\mathrm{CPh}}$ & 43.06 & 41.0 & 38.2 \\
\hline $\mathrm{H}_{\underline{C O C H}}$ & 73.06 & 72.5 & 71.7 \\
\hline $\mathrm{O}_{\mathbf{C H}} \mathrm{H}_{3}$ & 57.79 & 57.2 & 58.2 \\
\hline $\mathbf{C}_{\mathrm{q}}$ & 37.32 & 37.8 & 32.3 \\
\hline \multicolumn{4}{|c|}{${ }^{29}$ Si NMR Chemical Shifts } \\
\hline & $\begin{array}{l}-15.2 \\
{[\mathrm{~d}, \mathrm{~J}=7 \mathrm{~Hz}]}\end{array}$ & $\begin{array}{l}-16 \\
{[\mathrm{~d}, \mathrm{~J}=\sim 6 \mathrm{~Hz}]}\end{array}$ & -16 \\
\hline$\underline{\mathrm{SiC}}=\mathrm{CH}$ & $\begin{array}{l}-3.9 \\
{[\mathrm{~d}, \mathrm{~J}=29 \mathrm{~Hz}]}\end{array}$ & $\begin{array}{l}-12 \\
{[\mathrm{~d}, \mathrm{~J}=29 \mathrm{~Hz}]}\end{array}$ & $\begin{array}{l}-14 \\
{[\mathrm{~d}, \mathrm{~J}=28 \mathrm{~Hz}]}\end{array}$ \\
\hline
\end{tabular}

a from reference 1 


\section{Compound 7}

Yield $=8 \%,{ }^{1} \mathrm{H} \mathrm{NMR}^{1,2}\left(\mathrm{C}_{6} \mathrm{D}_{6}, 600 \mathrm{MHz}\right) \delta 8.16(\mathrm{~d}, J=7.6 \mathrm{~Hz}, 2 \mathrm{H}, o-\mathrm{Ph}-\mathrm{H}), 7.30(\mathrm{t}, J=7.8 \mathrm{~Hz}$, $m$-Ph-H) and 7.29 (d, $J=1.6 \mathrm{~Hz}, m-\mathrm{Tip}-\mathrm{H})(3 \mathrm{H}$ total), 7.12 (d, $J=16 \mathrm{~Hz}, \mathrm{Si}-\mathrm{CH}=\mathrm{C}), 7.11$ (d, $J=$ 1.6 Hz, m-Tip-H), 7.04 (d, $J=1.6 \mathrm{~Hz}, 1 \mathrm{H}, m$-Tip-H), 7.00 (d, $J=1.6 \mathrm{~Hz}, m$-Tip-H), 6.93 (d, $J=$ $1.6 \mathrm{~Hz}, 1 \mathrm{H}, m-\mathrm{Tip}-\mathrm{H}), 6.73$ (d, $J=16 \mathrm{~Hz}, 1 \mathrm{H}, \mathrm{C}=\mathrm{CH}-\mathrm{C}$ ), 6.58 (d, $J=1.6 \mathrm{~Hz}, 1 \mathrm{H}, m-\mathrm{Tip}-\mathrm{H}$ ), $4.93(\mathrm{~d}, J=10 \mathrm{~Hz}, 1 \mathrm{H}, \mathrm{CH}-\mathrm{Ph}), 4.66$ (s, $1 \mathrm{H}, \mathrm{C}=\mathrm{CH}_{2}$ ), 4.48 (s, $1 \mathrm{H}, \mathrm{C}=\mathrm{CH}_{2}$ ), 4.36 (sept, $J=6.6$ Hz, 1 H, iPr CH), 4.08 (d, $J=10$ Hz, 1 H, CH-OMe), 3.88 (sept, $J=6.6$ Hz, 1 H, iPr CH), 3.62 (sept, $J=6.6 \mathrm{~Hz}, 1 \mathrm{H}$, $i \operatorname{Pr} \mathrm{CH}$ ), 3.56 (m, $i \operatorname{Pr} \mathrm{CH}$ ), 3.34 (sept, $J=6.6 \mathrm{~Hz}, 1 \mathrm{H}, i \operatorname{Pr} \mathrm{CH}$ ), 2.82-2.70 (m, 3 H, iPr CH), 2.61 (sept, $J=6.6$ Hz, 1 H, iPr CH), 2.20 (s, 3 H, OCH OC 1.65 (d, J = 6.4 Hz, iPr $\left.\mathrm{CH}_{3}\right)$ and $1.62\left(\mathrm{~d}, J=6.4 \mathrm{~Hz}, 3 \mathrm{H}, i \operatorname{Pr~} \mathrm{CH}_{3}\right)\left(6 \mathrm{H}\right.$ total), 1.47 (d, $\left.J=6.4 \mathrm{~Hz}, i \operatorname{Pr~CH} \mathrm{CH}_{3}\right)$ and 1.46 (d, $\left.J=6.4 \mathrm{~Hz}, i \operatorname{Pr~} \mathrm{CH}_{3}\right)$ (6 H total), 1.34 (d, $\left.J=6.4 \mathrm{~Hz}, 3 \mathrm{H}, i \operatorname{Pr~} \mathrm{CH}_{3}\right), 1.28$ (d, $\left.J=6.4 \mathrm{~Hz}, i \operatorname{Pr} \mathrm{CH}_{3}\right)$, 1.24 (d, $\left.J=6.4 \mathrm{~Hz}, 3 \mathrm{H}, i \operatorname{Pr} \mathrm{CH}_{3}\right), 1.18$ (d, $\left.J=6.4 \mathrm{~Hz}, i \operatorname{Pr} \mathrm{CH}_{3}\right), 1.17$ (d, $\left.J=6.4 \mathrm{~Hz}, i \operatorname{Pr} \mathrm{CH}_{3}\right), 1.10$ (d, $\left.J=6.4 \mathrm{~Hz}, i \operatorname{Pr} \mathrm{CH}_{3}\right)$ and $1.08(\mathrm{~d}, J=6.4 \mathrm{~Hz}, i \operatorname{Pr~CH} 3)(6 \mathrm{H}$ total), $1.02(\mathrm{~d}, J=6.4 \mathrm{~Hz}, 3 \mathrm{H}, i \mathrm{Pr}$ $\mathrm{CH}_{3}$ ), 0.79 (d, $J=6.4 \mathrm{~Hz}, 3 \mathrm{H}, i \operatorname{Pr} \mathrm{CH}_{3}$ ), 0.65 (d, $J=6.4 \mathrm{~Hz}, 3 \mathrm{H}, i \operatorname{Pr} \mathrm{CH}_{3}$ ), 0.61 (d, $J=6.4 \mathrm{~Hz}, 3$ H, $i \operatorname{Pr~} \mathrm{CH}_{3}$ ), 0.48 (d, $J=6.4 \mathrm{~Hz}, 3 \mathrm{H}, i \operatorname{Pr~} \mathrm{CH}_{3}$ ), 0.36 (d, $J=6.4 \mathrm{~Hz}, 3 \mathrm{H}, i \operatorname{Pr~CH}$ ), 0.15 (d, $J=6.4$ $\left.\mathrm{Hz}, 3 \mathrm{H}, \mathrm{iPr} \mathrm{CH}_{3}\right) .{ }^{13} \mathrm{C}$ NMR $\left(151 \mathrm{MHz}, \mathrm{C}_{6} \mathrm{D}_{6}\right) \delta 155.57,155.31,154.76,154.27,154.16,152.76$, 149.41, 149.15, 148.13 (all Tip-oC and Tip-pC), 145.21 (Ph), 145.1 (br, =C x3), 143.44 (Ph-iC), 141.27 (Ph), 139.73, 138.48, 137.93 (all Tip-iC), 135.08 (br, Ph-oC), 133.2 (=C), 127.98 (Ph-mC), 127.77 (Ph-pC), 125.67 (Ph), 125.18 (br, Ph), 124.7, 123.9, 123.2, 122.6, 122.5, 120.0 (all TipmC), 91.24 (CHOMe), 57.42 (OMe), 42.30 (CHPh) 37.53, 37.52, 35.5, 35.3, 34.28, 34.27, 34.26, 33.8, 30.5 (all $i \mathrm{Pr} \mathrm{CH}$ ) 30.4, 27.1, 26.9, 26.4, 25.9, 25.7, 25.4, 25.3, 24.5, 24.0, 23.83 (x2), 23.79

\footnotetext{
${ }^{1}$ Signals for the second phenyl group could not be assigned with confidence

2 Integration values only shown for isolated signals. Overlapping signals are assigned by 2D NMR spectroscopy but not integrated.
} 
(x2), 23.9, 23.6, 23.1, 20.7 (all $\left.i \mathrm{Pr} \mathrm{CH}_{3}\right) .{ }^{29} \mathrm{Si}^{-1}{ }^{1} \mathrm{H}\left(\mathrm{C}_{6} \mathrm{D}_{6}\right) \delta$-12 (SiTip2), -32 (SiPhTip). ESI-MS: m/z 951.6279 (calcd for $\mathrm{C}_{64} \mathrm{H}_{88} \mathrm{NaOSi}_{2}$, 951.6271).

Figure S1: a) ${ }^{1} \mathrm{H}$ NMR spectrum (600 MHz, $\mathrm{C}_{6} \mathrm{D}_{6}$ ) of 7; b) Expansion from 6.70 - 7.12 ppm; c) Expansion from 3.8-5.0 ppm

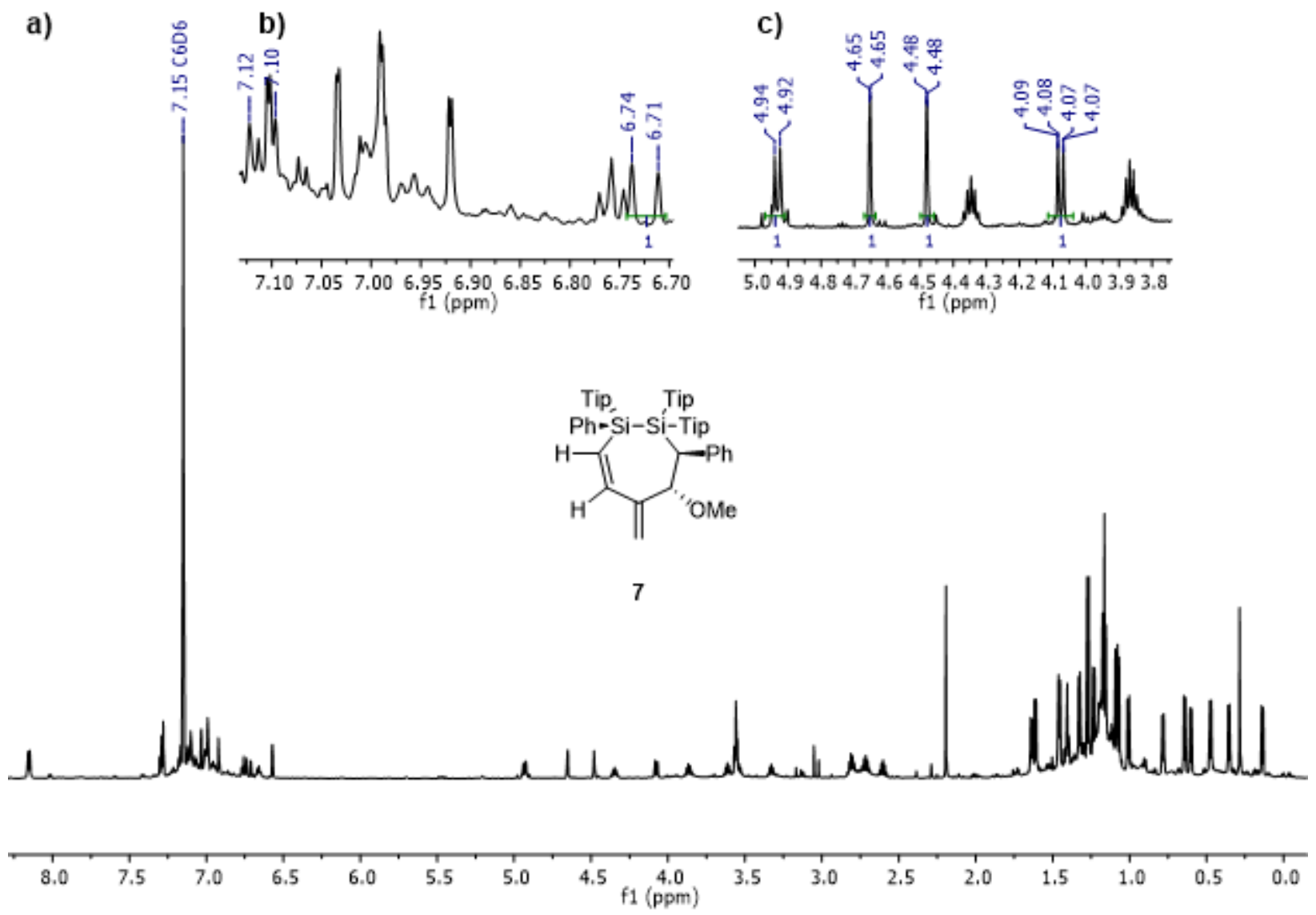


Figure S2: ${ }^{1} \mathrm{H}-{ }^{-1} \mathrm{H}$ gCOSY NMR spectrum of $7\left(600 \mathrm{MHz}, \mathrm{C}_{6} \mathrm{D}_{6}\right)$.

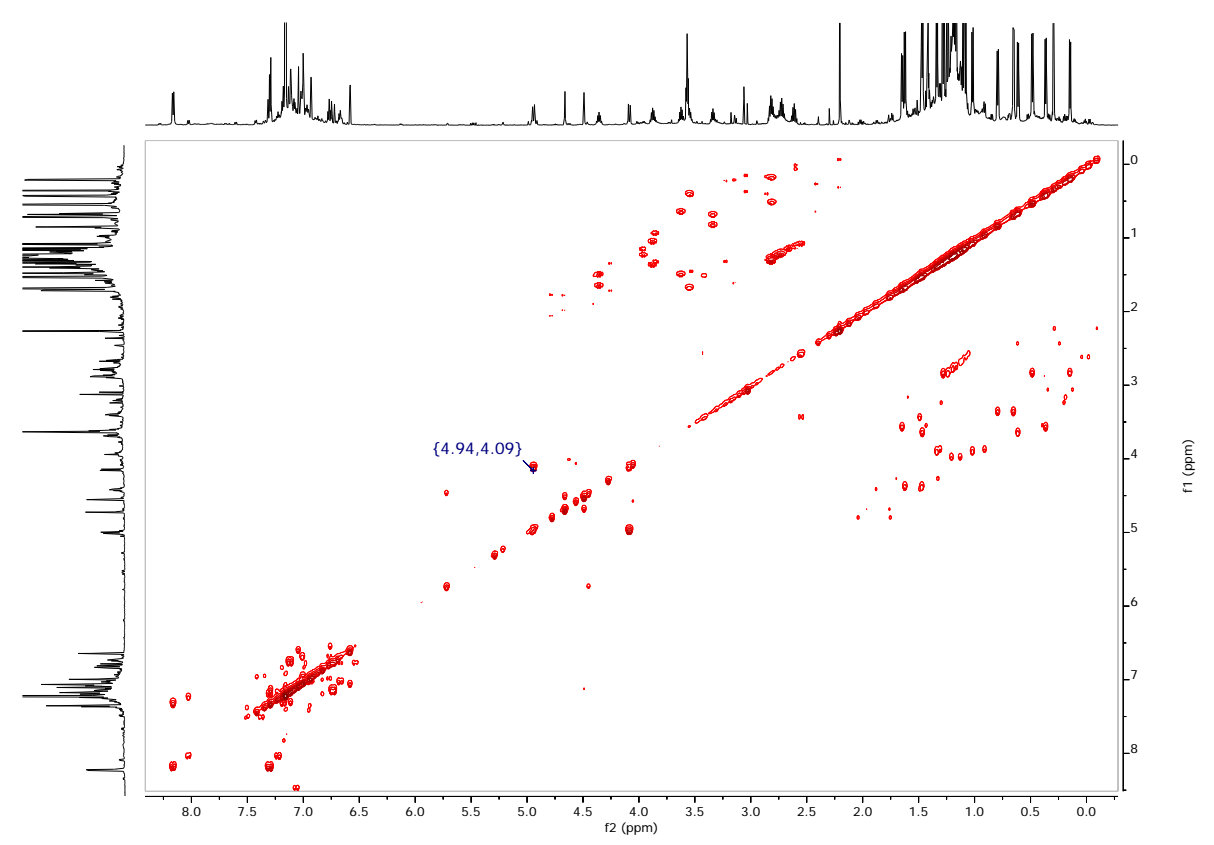

Figure S3: Expansion of the ${ }^{13} \mathrm{C}-{ }^{1} \mathrm{H}$ gHSQCAD NMR spectrum of $7\left(600 \mathrm{MHz}, \mathrm{C}_{6} \mathrm{D}_{6}\right)$ with ${ }^{1} \mathrm{H} ;{ }^{13} \mathrm{C}$ ranges of: $(0.0-1.8 \mathrm{ppm} ; 20-31 \mathrm{ppm})(2.0-5.0 \mathrm{ppm} ; 30-60 \mathrm{ppm})(4.0-4.8 \mathrm{ppm} ; 90-125$ ppm) (6.6 - $8.2 \mathrm{ppm} ; 120$ - $145 \mathrm{ppm})$
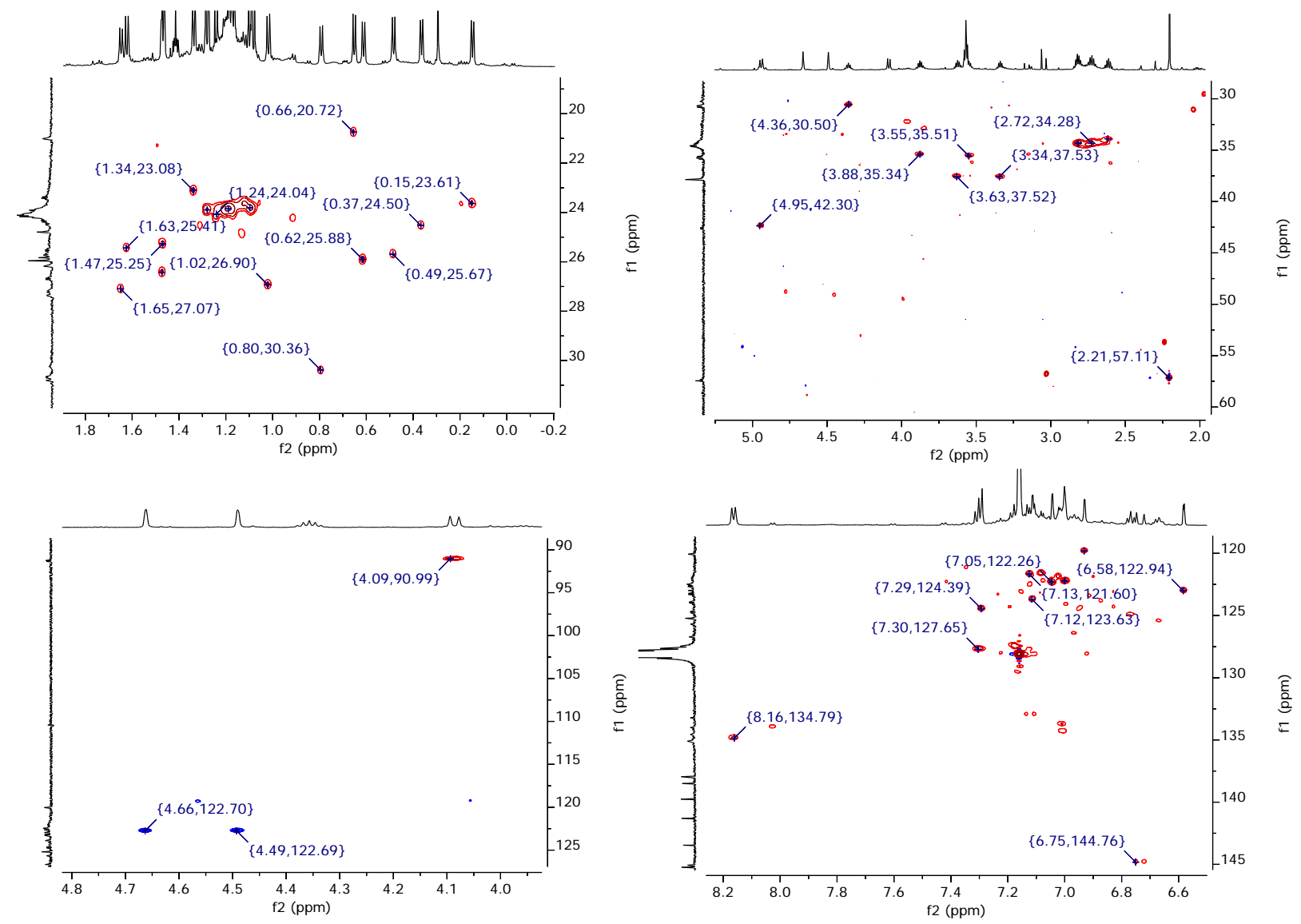
Figure S4: Expansion of the ${ }^{13} \mathrm{C}-{ }^{1} \mathrm{H}$ gHMBC NMR spectrum of 7 (600 MHz, $\left.\mathrm{C}_{6} \mathrm{D}_{6}\right)$ with ${ }^{1} \mathrm{H} ;{ }^{13} \mathrm{C}$ ranges of: $(0.0-1.7 \mathrm{ppm} ; 148$ - $157 \mathrm{ppm})(4.45$ - $4.80 \mathrm{ppm} ; 144-147 \mathrm{ppm})$ (6.55 - 7.35 ppm; 119 - 146 ppm) (8.12 - 8.21 ppm; 125 - 140 ppm)

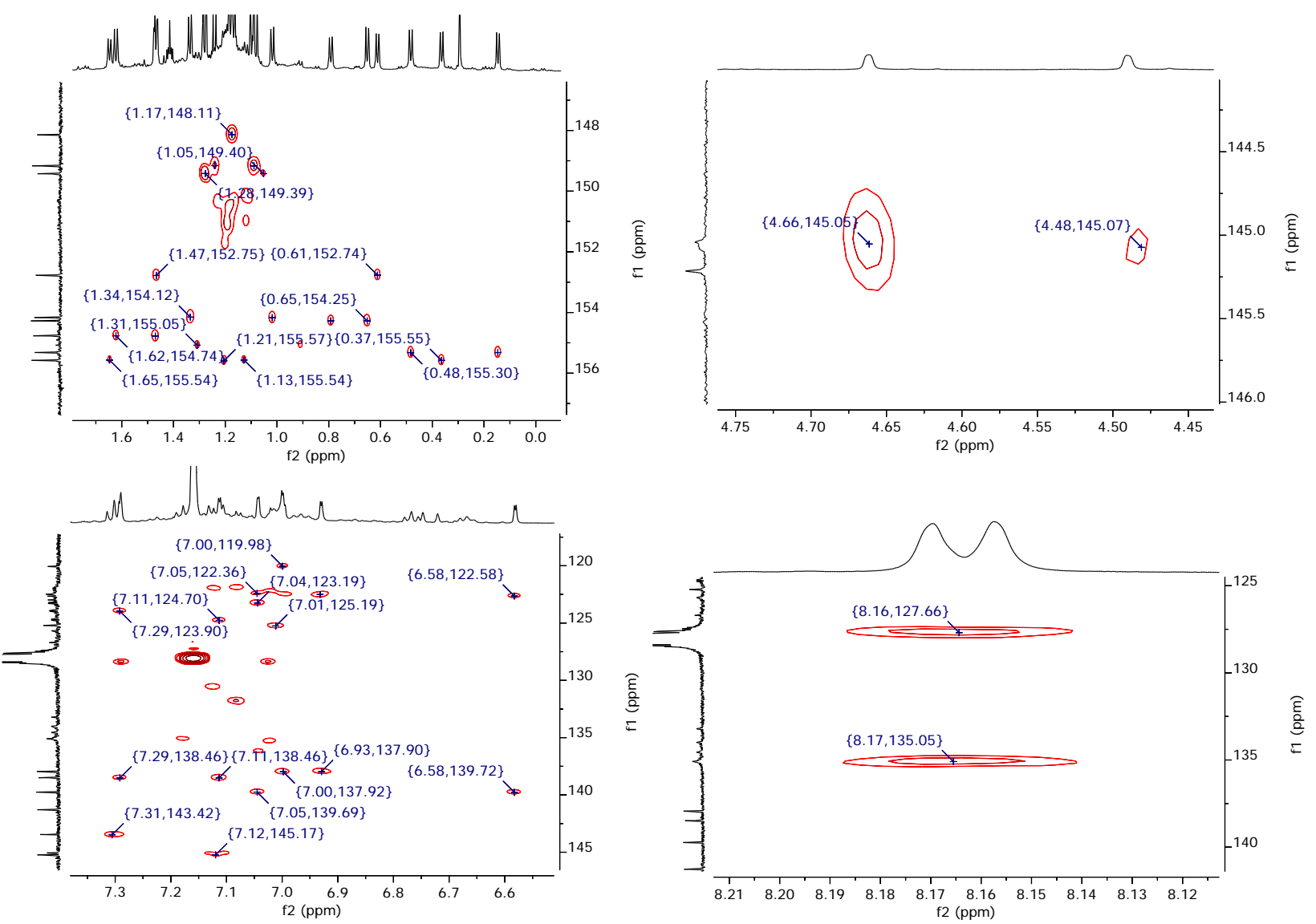


Figure S5: ${ }^{29} \mathrm{Si}-{ }^{1} \mathrm{H}$ gHMBC NMR spectrum of 7 (600 MHz, $\left.\mathrm{C}_{6} \mathrm{D}_{6}\right)$

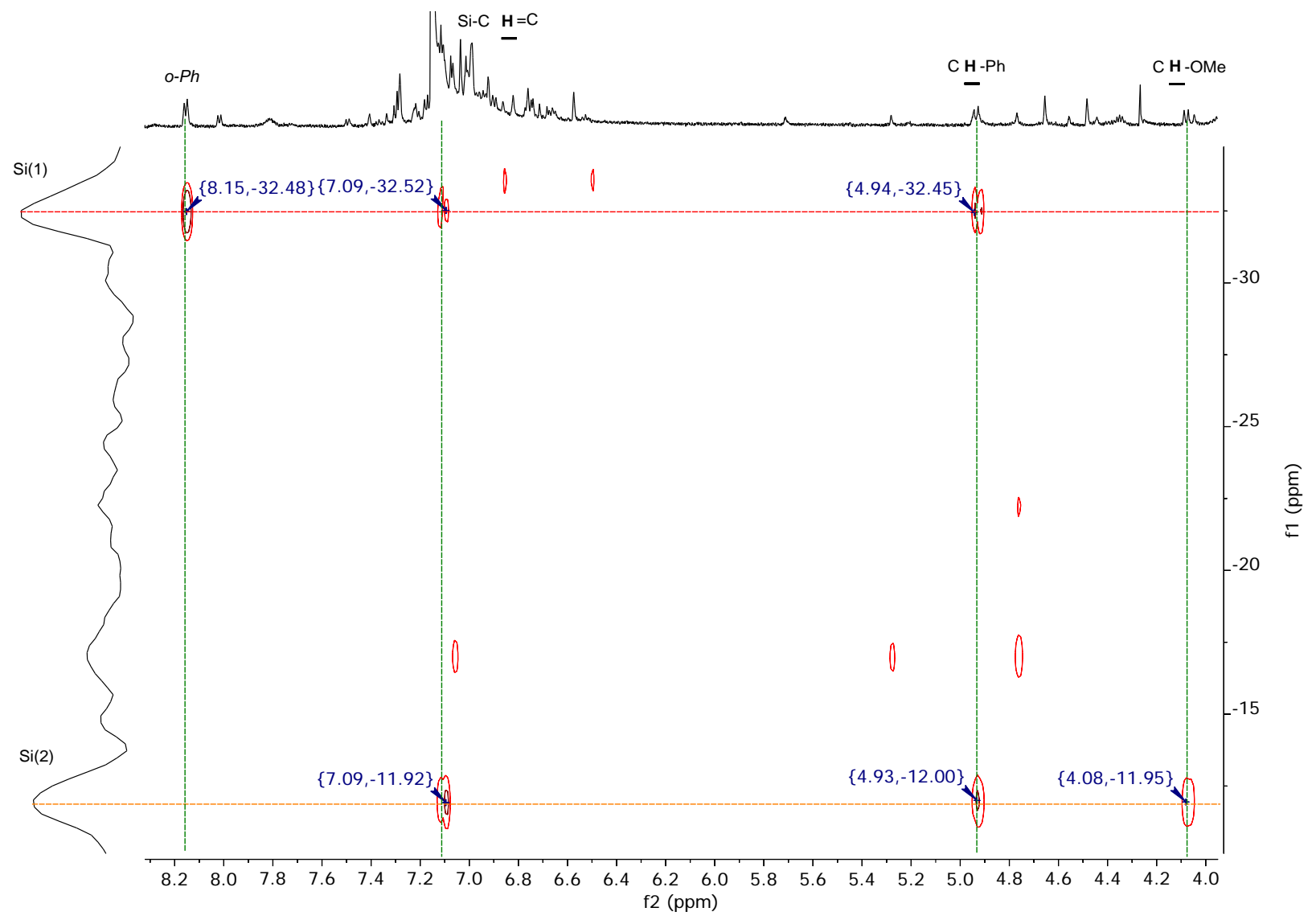




\section{Compound 8}

Yield $=8 \%,{ }^{1} \mathrm{H}$ NMR $\left(\mathrm{C}_{6} \mathrm{D}_{6}, 600 \mathrm{MHz}\right)^{3} \delta 8.28(\mathrm{~d}, 1 \mathrm{H}, \mathrm{Si}-o-\mathrm{Ph}-\mathrm{H}), 7.6,7.3,7.2(\mathrm{Si}-\mathrm{Ph}-\mathrm{H})^{4}, 7.6$, 7.4, 7.0, 6.7, 6.1 (C-Ph-H) ${ }^{2}, 7.36$ (d, $J=1.9$ Hz, m-Tip-H), 7.27 (m, m-Tip-H), 7.10 (s, m-Tip-H), 7.06 (s, m-Tip-H), 6.93 (d, $J=1.9 \mathrm{~Hz}, m$-Tip-H), 6.90 (d, $J=1.9 \mathrm{~Hz}, 1 \mathrm{H}, m$-Tip-H), 6.71 (q, $J=$ 3.5 Hz, 1 H, Si-CH=C), 4.75 (sept, $J=6.4$ Hz, 1 H, iPr CH), 4.02 (s, 1 H, CH-Ph), 3.93 - 3.80 (m, iPr CH x2), 3.71 (s, 1 H, CH-OMe), 3.61 (sept, $J=6.4$ Hz, 1 H, iPr CH), 3.36 - 3.22 (m, iPr CH x2), 3.15 (m, iPr CH), 3.02 (s, 3 H, $\mathrm{OCH}_{3}$ ), 2.76 (m, iPr CH), 2.52 (sept, $J=6.4$ Hz, $1 \mathrm{H}, i \operatorname{Pr} \mathrm{CH}$ ), $1.74\left(\mathrm{~d}, J=6.9 \mathrm{~Hz}, 3 \mathrm{H}, i \operatorname{Pr} \mathrm{CH}_{3}\right), 1.61\left(\mathrm{~d}, J=6.9 \mathrm{~Hz}, 3 \mathrm{H}, i \operatorname{Pr} \mathrm{CH}_{3}\right), 1.55$ (d, $J=6.9 \mathrm{~Hz}, 3 \mathrm{H}, i \operatorname{Pr}$ $\mathrm{CH}_{3}$ ), 1.47 (m, $i \operatorname{Pr} \mathrm{CH}_{3}$ ), 1.43 (d, $J=6.9 \mathrm{~Hz}, 3 \mathrm{H}$, $\left.i \operatorname{Pr} \mathrm{CH}_{3}\right), 1.37$ (m, $i \mathrm{Pr} \mathrm{CH}_{3}$ ), 1.33 (s, $\left.\mathrm{CH}_{3}\right), 1.30$

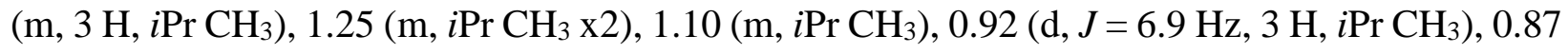
(d, $J=6.9 \mathrm{~Hz}, i \operatorname{Pr} \mathrm{CH}_{3}$ ), 0.69 (d, $J=6.9 \mathrm{~Hz}, 3 \mathrm{H}, i \operatorname{Pr~CH}$ ), 0.66 (m, $\left.i \operatorname{Pr} \mathrm{CH}_{3}\right), 0.65$ (m, $\left.i \operatorname{Pr~} \mathrm{CH}_{3}\right)$, 0.34 (d, $J=6.9 \mathrm{~Hz}, 3 \mathrm{H}, i \operatorname{Pr~CH}$ ), 0.30 (d, $\left.J=6.9 \mathrm{~Hz}, i \operatorname{Pr} \mathrm{CH}_{3}\right), 0.20$ (d, $\left.J=6.9 \mathrm{~Hz}, 3 \mathrm{H}, i \operatorname{Pr~} \mathrm{CH}_{3}\right)$. ${ }^{13} \mathrm{C}$ NMR $\left(\mathrm{C}_{6} \mathrm{D}_{6}\right) \delta=218.4$ (allene-C),159.5, 156.8, 155.9 (x2), 154.6, 154.6, 153.2, 152.4, 149.2 (all Tip-oC and Tip-pC), 141.5 (iTip), 140.1 (Ph-oC) 139.7 (iTip), 137.9 (iTip), 124.2, 123.8, 123.7, 122.7, 122.3, 121.6 (all Tip-mC), 96.8 (C=CMe), 92.3 (Si-CH=C), 91.7 (CH-OMe), 56.8 $\left(\mathrm{OCH}_{3}\right)$, 54.1 (CH-Ph), 37.7, 37.6, 35.2, 35.2 (x2), 34.9, 34.3, 34.0, 33.8 (all iPr CH), 29.1, 29.0, 28.0, 27.6, 26.4, 26.3, 26.0, 25.5 (x2), 25.0, 24.9, 24.6, 24.1, 24.0 (x2), 23.2, 21.9 (all iPr $\mathrm{CH}_{3}$ ), $17.6\left(\mathrm{CH}_{3}\right) .{ }^{29} \mathrm{Si}^{-1} \mathrm{H}$ gHMBC NMR (C6 $\left.\mathrm{D}_{6}, 119 \mathrm{MHz}\right): \delta=-28$ (SiTip $)$ ), -31 (SiPhTip). ESI-MS: m/z 951.6272 (calcd for $\mathrm{C}_{64} \mathrm{H}_{88} \mathrm{NaOSi}_{2}, 951.6271$ ).

\footnotetext{
${ }^{3}$ Integration values only shown for isolated signals. Overlapping signals are assigned by 2D NMR spectroscopy but not integrated.

${ }^{4} \mathrm{Ph}-\mathrm{H}$ chemical shifts were extracted from the ${ }^{1} \mathrm{H}-{ }^{1} \mathrm{H}$ COSY spectrum
} 
Figure S6: a) ${ }^{1} \mathrm{H}$ NMR spectrum of $8\left(600 \mathrm{MHz}, \mathrm{C}_{6} \mathrm{D}_{6}\right)$; b) Expansion from 6.69 - $6.71 \mathrm{ppm}$; c) Expansion from 3.6 - 4.1 ppm; d) Expansion from 1.30 - 1.35 ppm
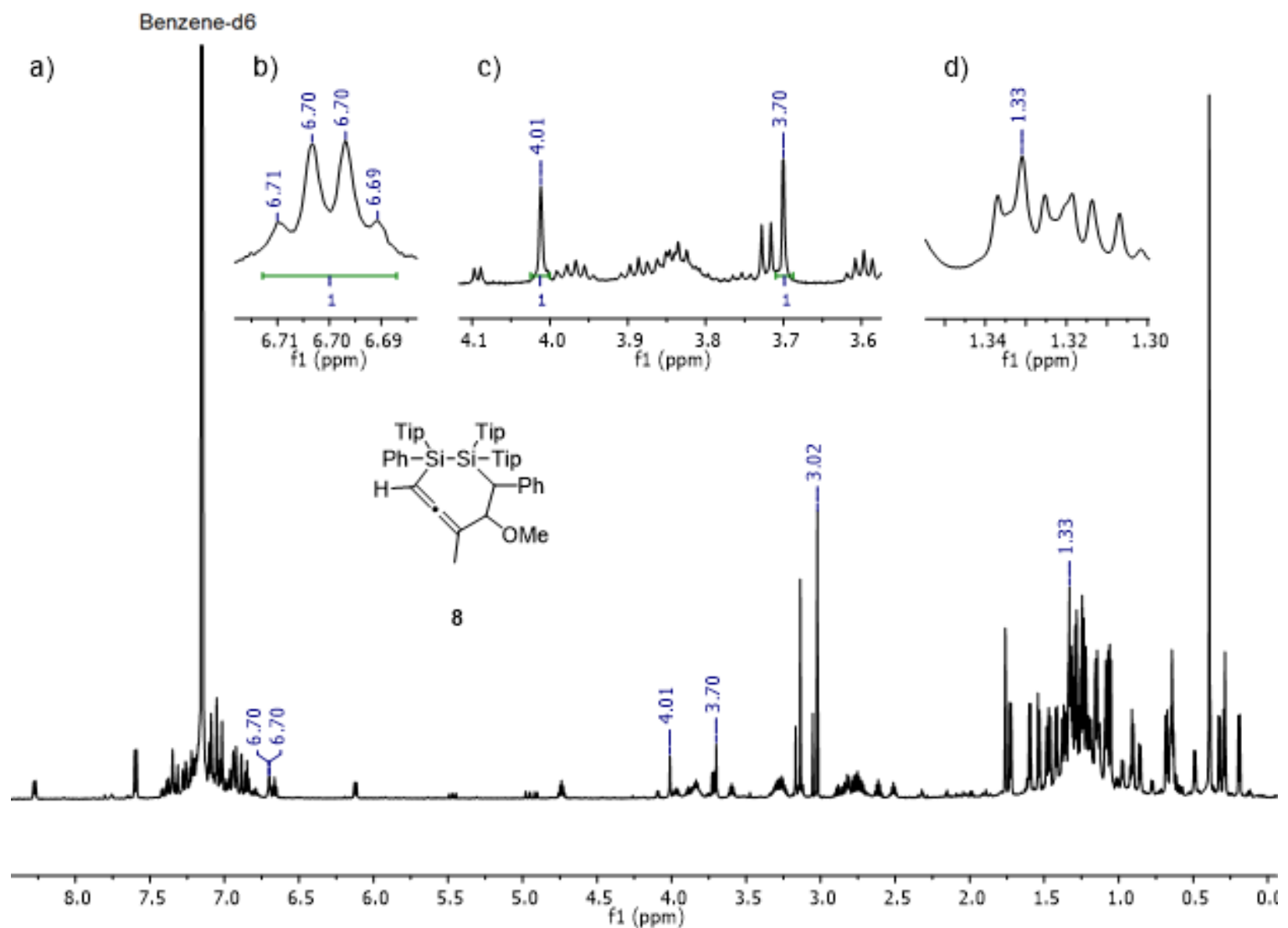
Figure S7: Expansion of the ${ }^{1} \mathrm{H}-{ }^{1} \mathrm{H}$ COSY NMR spectrum of $8\left(600 \mathrm{MHz}, \mathrm{C}_{6} \mathrm{D}_{6}\right)$ illustrating correlations among the Si-Ph hydrogens (f1: 6.5 - $8.5 \mathrm{ppm}$; $\mathrm{f} 2: 6.6-8.3 \mathrm{ppm}$ ), the C-Ph hydrogens (f1: $6.0-7.4 \mathrm{ppm}$; f2: $6.1-7.7 \mathrm{ppm}$ ), and the correlation between the $\mathrm{C} \underline{\mathrm{H}}-\mathrm{OMe}$ and $\mathrm{C} \underline{\mathrm{H}}-\mathrm{Ph}$ hydrogens $(\mathrm{f} 1=\mathrm{f} 2: 3.4-4.3 \mathrm{ppm})$,
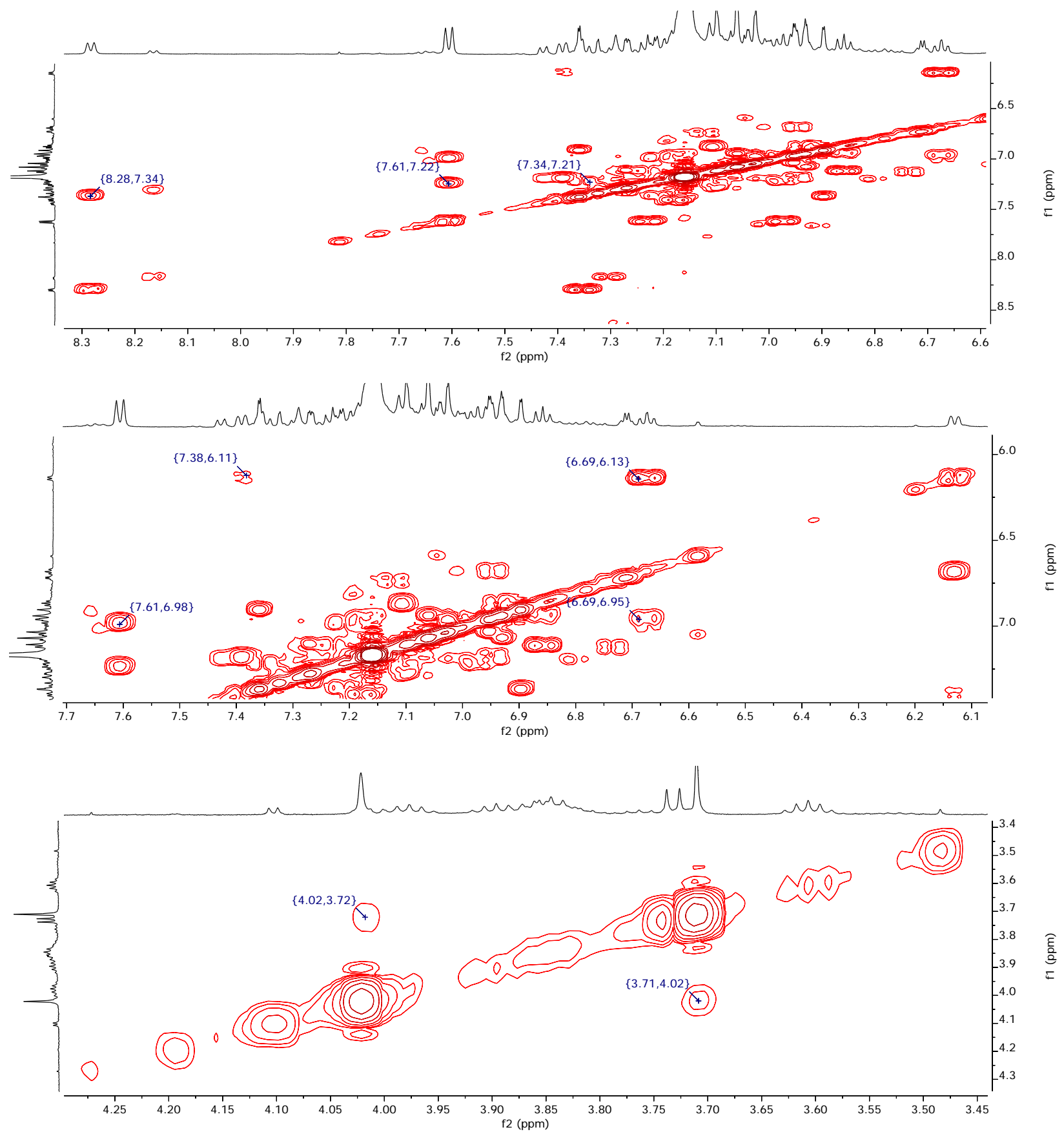
Figure S8: Expansion of the ${ }^{13} \mathrm{C}-{ }^{1} \mathrm{H}$ gHSQCAD NMR spectrum of $8\left(600 \mathrm{MHz}, \mathrm{C}_{6} \mathrm{D}_{6}\right)$ with ${ }^{1} \mathrm{H} ;{ }^{13} \mathrm{C}$ ranges of $(0.0-1.9 \mathrm{ppm} ; 17-32 \mathrm{ppm})(2.5-4.9 \mathrm{ppm} ; 30-55 \mathrm{ppm})(6.0-8.3 \mathrm{ppm} ; 120-141$ ppm) (3.7 - 6.8 ppm; $91-93$ ppm)
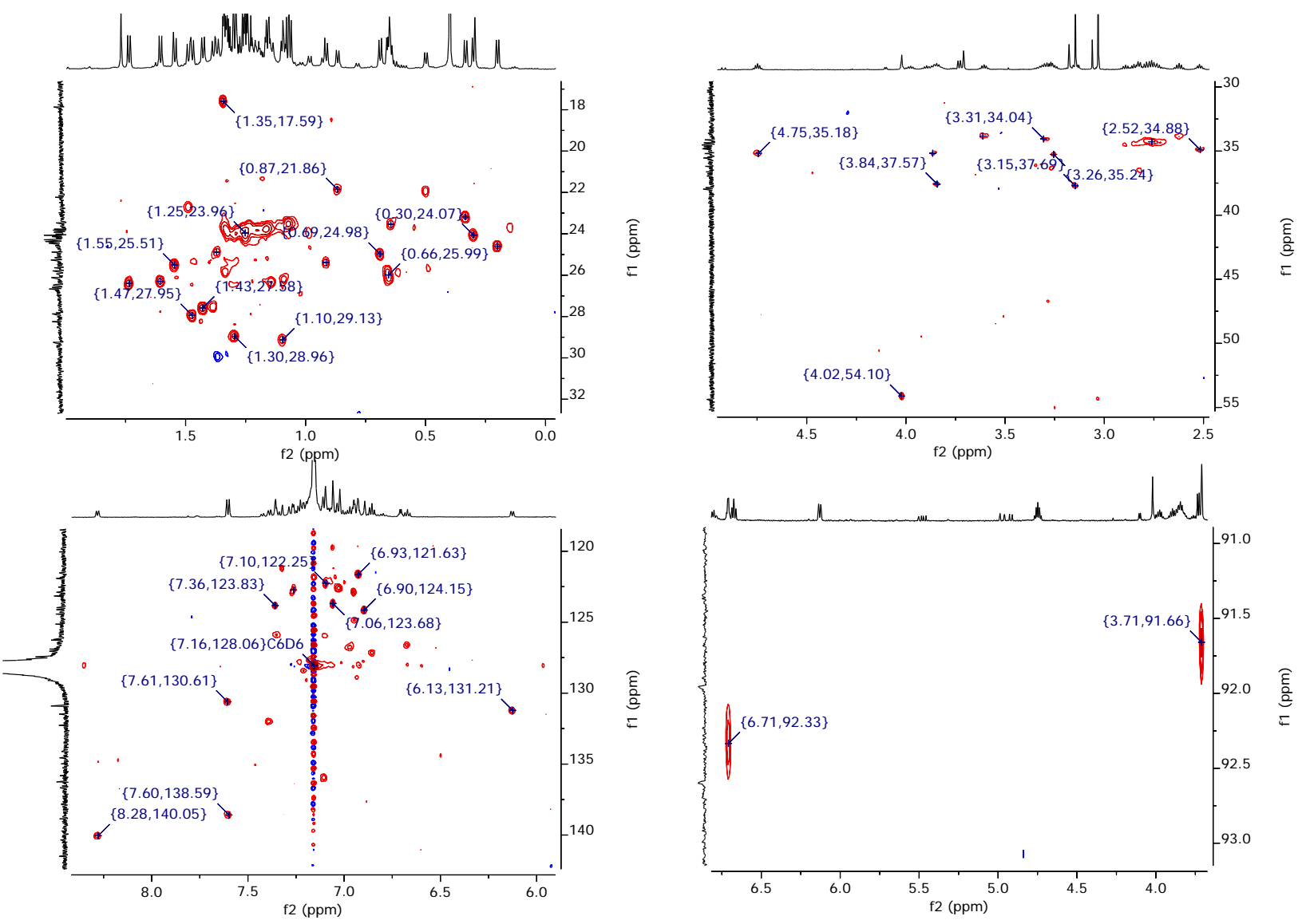
Figure S9: Expansions of the ${ }^{13} \mathrm{C}-{ }^{1} \mathrm{H}$ gHMBC NMR spectrum of $8\left(600 \mathrm{MHz}, \mathrm{C}_{6} \mathrm{D}_{6}\right)$ with ${ }^{1} \mathrm{H} ;{ }^{13} \mathrm{C}$ ranges of: (0.0 - 2.0 ppm; 149 - 158 ppm) (3.69 - 3.74 ppm; 217.5 - 219.0 ppm) (6.7 - 8.4 ppm, $120-150 \mathrm{ppm})$
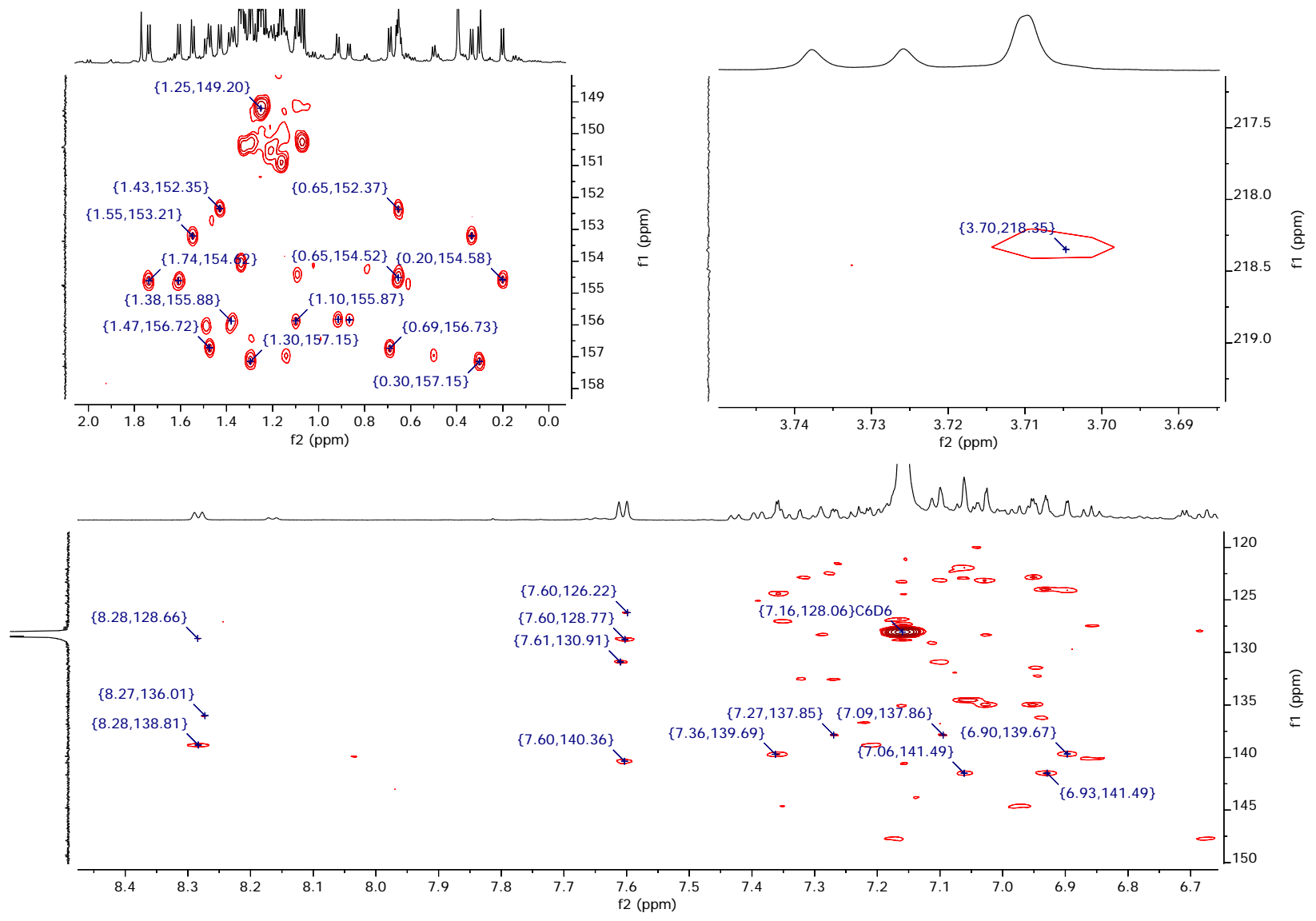
Figure S10: ${ }^{29} \mathrm{Si}-{ }^{1} \mathrm{H}$ gHMBC NMR spectrum of $8\left(600 \mathrm{MHz}, \mathrm{C}_{6} \mathrm{D}_{6}\right)$

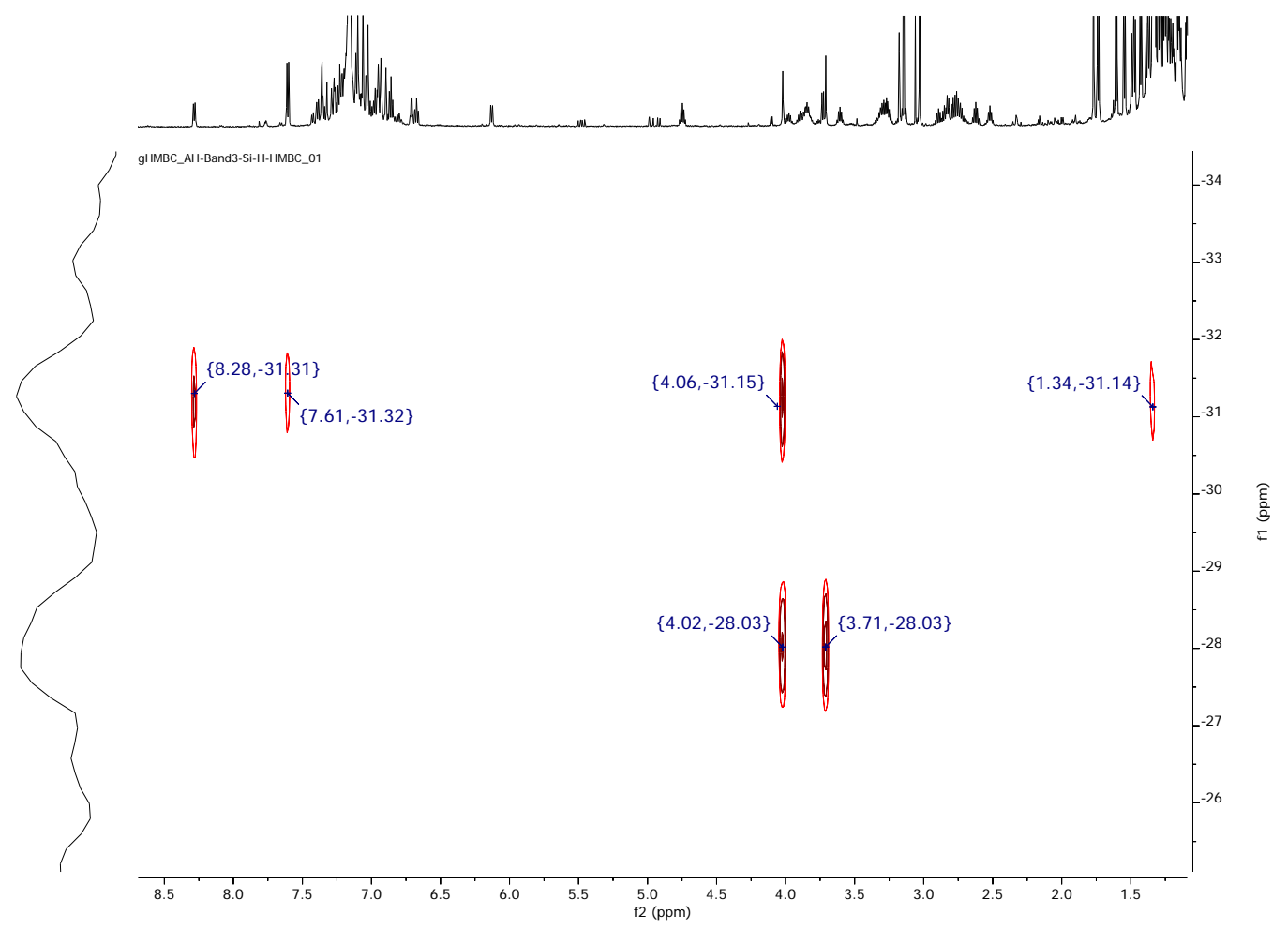




\section{Compound 9}

Yield $=15 \%{ }^{1} \mathrm{H}$ NMR $\left(\mathrm{C}_{6} \mathrm{D}_{6}, 600 \mathrm{MHz}\right)^{5} \delta 7.46(\mathrm{~s}, 1 \mathrm{H}, \mathrm{Si}-\mathrm{CH}=\mathrm{C}), 7.40(\mathrm{~d}, J=1.5 \mathrm{~Hz}, 1 \mathrm{H}, m-$

Tip-H), 7.34 (d, J = 1.5 Hz, 1 H, m-Tip-H), 7.27 (d, J = 1.5 Hz, 1 H, m-Tip-H), 7.26 - 7.23 (m, 2 H, o-Ph-H), 7.13 - 7.00 (m, Ph), 7.11 (d, $J=1.5$ Hz, m-Tip-H) (6 H total), 7.03 (d, $J=1.5 \mathrm{~Hz}, 1$ H, m-Tip-H), 7.00 (d, $J=1.5$ Hz, 1 H, m-Tip-H), 6.9-6.8 (m, 3 H, Ph), 4.04 (sept, $J=6.5$ Hz, 1 H, iPr CH), 3.99 (sept, $J=6.5 \mathrm{~Hz}, 1 \mathrm{H}, i \operatorname{Pr} \mathrm{CH}$ ), 3.93 (sept, $J=6.5 \mathrm{~Hz}, 1 \mathrm{H}, i \operatorname{Pr} \mathrm{CH}), 3.60$ (d, $J=4.7$ Hz, 1 H, CH-OMe), 3.34-3.24 (m, 3 H, iPr CH), 3.13 (s, 3 H, OCH $), 2.91$ (sept, $J=6.5$ Hz, 1 H, iPr CH), 2.84 (d, $J=4.1 \mathrm{~Hz}, 1 \mathrm{H}, \mathrm{CH}-\mathrm{Ph}), 2.74$ (sept, $J=6.5 \mathrm{~Hz}, 2 \mathrm{H}, i \operatorname{Pr} \mathrm{CH}), 1.60$ (d, $J=6.8$ Hz, $3 \mathrm{H}, o-i \operatorname{Pr~} \mathrm{CH}_{3}$ ), 1.58 (d, $J=6.8 \mathrm{~Hz}, 3 \mathrm{H}, o-i \operatorname{Pr~} \mathrm{CH}_{3}$ ), 1.54 (d, $J=6.8 \mathrm{~Hz}, 3 \mathrm{H}, o-i \mathrm{Pr} \mathrm{CH}_{3}$ ), $1.38\left(\mathrm{~d}, J=6.8 \mathrm{~Hz}, 3 \mathrm{H}, o-i \operatorname{Pr} \mathrm{CH}_{3}\right), 1.34\left(\mathrm{~d}, J=6.8 \mathrm{~Hz}, 1 o-\right.$ and $\left.4 p-i \mathrm{Pr} \mathrm{CH}_{3}\right), 1.25\left(\mathrm{~s}, 3 \mathrm{H}, \mathrm{CH}_{3}\right)$, 1.20 (d, $J=6.8 \mathrm{~Hz}, o-i \operatorname{Pr~CH}$ ), 1.17 and 1.16 (both d, $p$-iPr $\mathrm{CH}_{3}$ ), 1.02 (d, $J=6.8 \mathrm{~Hz}, 3 \mathrm{H}, o-i \operatorname{Pr}$ $\mathrm{CH}_{3}$ ), 0.98 (d, $J=6.8 \mathrm{~Hz}, 3 \mathrm{H}, o-i \operatorname{Pr} \mathrm{CH}_{3}$ ), 0.94 (d, $J=6.8 \mathrm{~Hz}, 3 \mathrm{H}, o-i \operatorname{Pr} \mathrm{CH}_{3}$ ), 0.83 (d, $J=6.8$ Hz, $3 \mathrm{H}, o-i \operatorname{Pr~} \mathrm{CH}_{3}$ ), 0.71 (d, $J=6.8 \mathrm{~Hz}, 3 \mathrm{H}, o-i \operatorname{Pr~} \mathrm{CH}_{3}$ ), 0.58 (d, $\left.J=6.8 \mathrm{~Hz}, 3 \mathrm{H}, o-i \mathrm{Pr} \mathrm{CH}_{3}\right)$. ${ }^{13} \mathrm{C}$ NMR $\left(\mathrm{C}_{6} \mathrm{D}_{6}\right) \delta 177.2$ (Si-C=CH), 156.8, 156.1 (x2), 155.7, 154.6, 154.2 (all Tip o-C), 150.5, 150.1(x2) (all Tip p-C), 152.3 (Si-CH=C), 137.8, 134.7, 133.0 (all Tip i-C), 139.9 (i-PhSi), 136.4 (o-PhSi), 129.2 (Ph), 129.1 (p-PhSi), 127.4 (m-PhSi), 122.9, 122.8, 122.7, 122.4, 121.2, 119.8 (all Tip m-C), 72.5 (CHOMe), 57.2 (OMe), 41.0 (CHPh) 37.1 ( CH $\left._{3} \mathrm{C}_{(\text {Cyclopropyl) }}\right)$ 36.6, 36.1, 35.5 (x2), 34.8 (x2), 34.5, 34.2, 34.1 (all $i \mathrm{Pr} \mathrm{CH}_{3}$ ), 29.4, 28.9, 27.6, 26.7, 26.6, 25.7, 25.1, 24.6, 24.3 (x2),

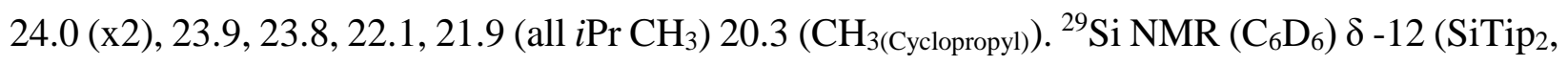
${ }^{3} J_{\mathrm{Si}-\mathrm{H}}=29 \mathrm{~Hz}$ ), -16 (SiPhTip). ESI-MS m/z 951.6250 (calcd for $\mathrm{C}_{64} \mathrm{H}_{88} \mathrm{NaOSi}_{2}, 951.6271$ ).

\footnotetext{
${ }^{5}$ Integration values only shown for isolated signals. Overlapping signals are assigned by 2D NMR spectroscopy but not integrated.
} 
Figure S11: a) ${ }^{1} \mathrm{H}$ NMR spectrum of 9 (600 MHz, $\left.\mathrm{C}_{6} \mathrm{D}_{6}\right)$; b) Expansion from 3.56 - 3.64 ppm; c) Expansion from $2.80-2.90$ ppm

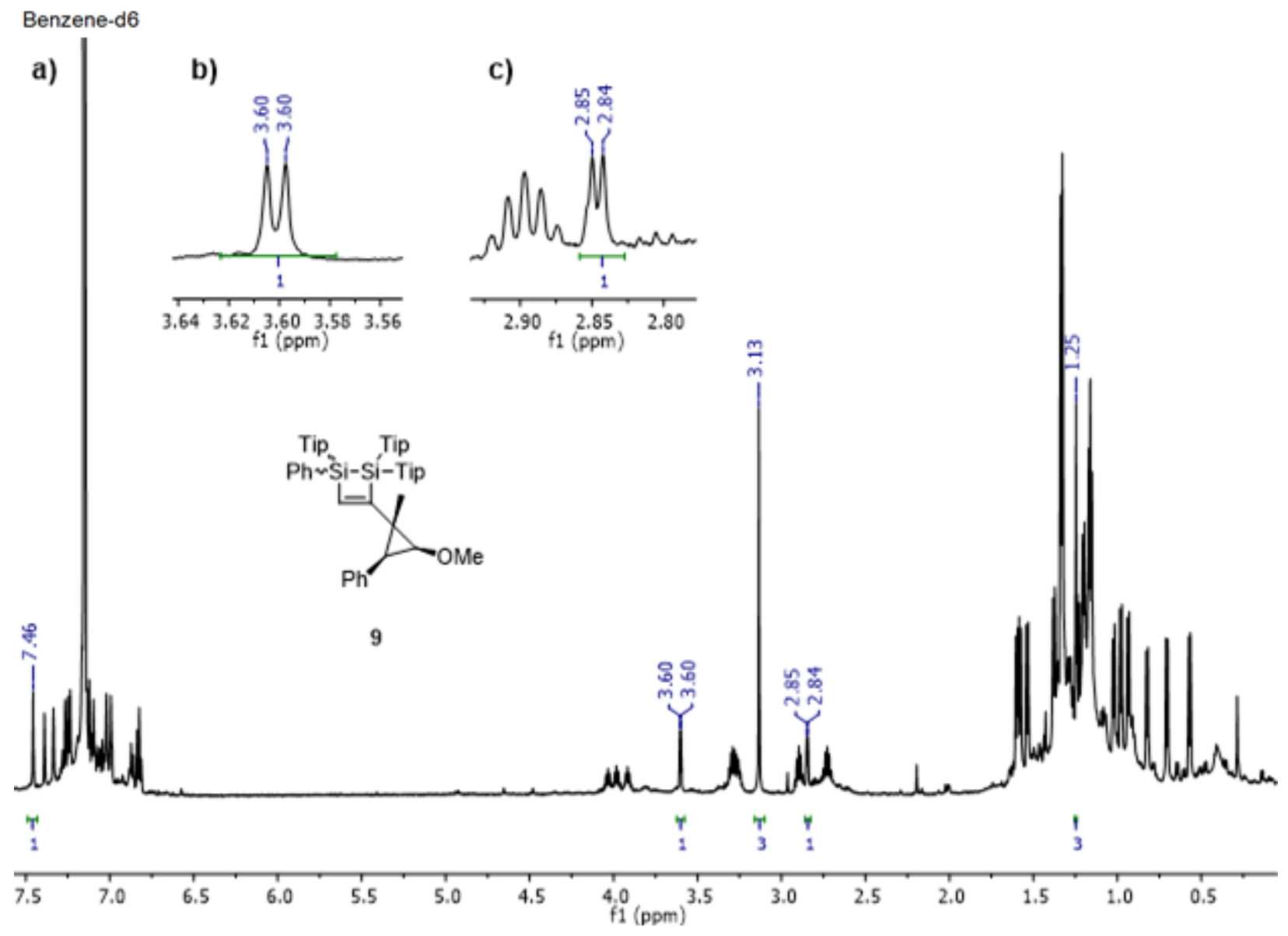


Figure S12: Expansion of the ${ }^{13} \mathrm{C}-{ }^{1} \mathrm{H}$ gHSQC NMR spectrum of $9\left(600 \mathrm{MHz}, \mathrm{C}_{6} \mathrm{D}_{6}\right)$ with ${ }^{1} \mathrm{H} ;{ }^{13} \mathrm{C}$ ranges of: (0.55 - 1.70 ppm; 20 - 30 ppm) (2.5 - 4.5 ppm; 30 - 75 ppm) (6.4 - 7.8 ppm; 118 $155 \mathrm{ppm})$

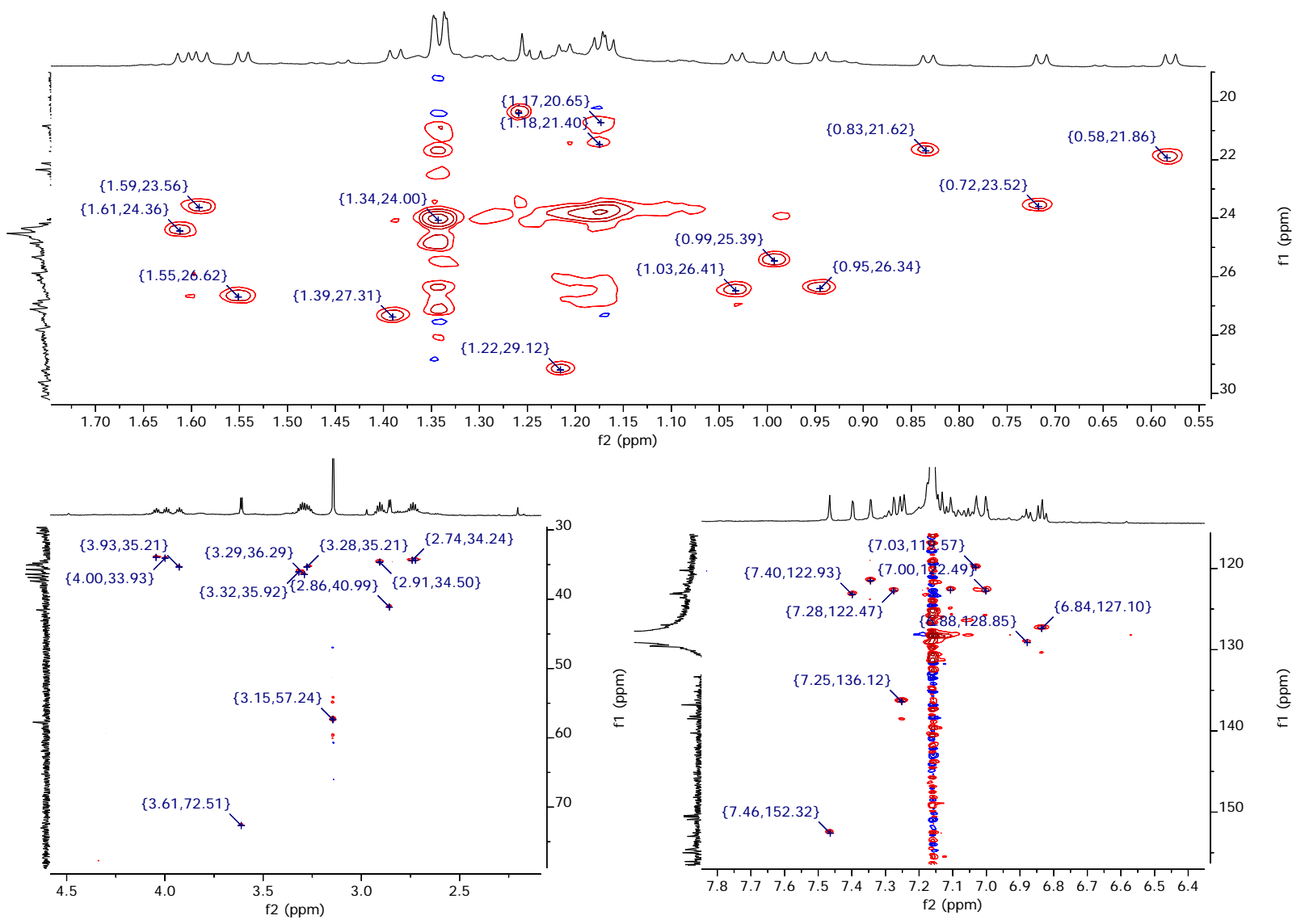


Figure S13: Expansion of the ${ }^{13} \mathrm{C}-{ }^{1} \mathrm{H}$ gHMBC NMR spectrum of $\mathbf{9}\left(600 \mathrm{MHz}, \mathrm{C}_{6} \mathrm{D}_{6}\right)$ with ${ }^{1} \mathrm{H} ;{ }^{13} \mathrm{C}$ ranges of: (0.0 - 2.0 ppm; 20 - 42 ppm) (6.7 - 7.6 ppm; 33 - 38 ppm) (6.0 - 8.2 ppm; 120 - 180 ppm) (0.5 - 1.9 ppm; $150-178$ ppm)
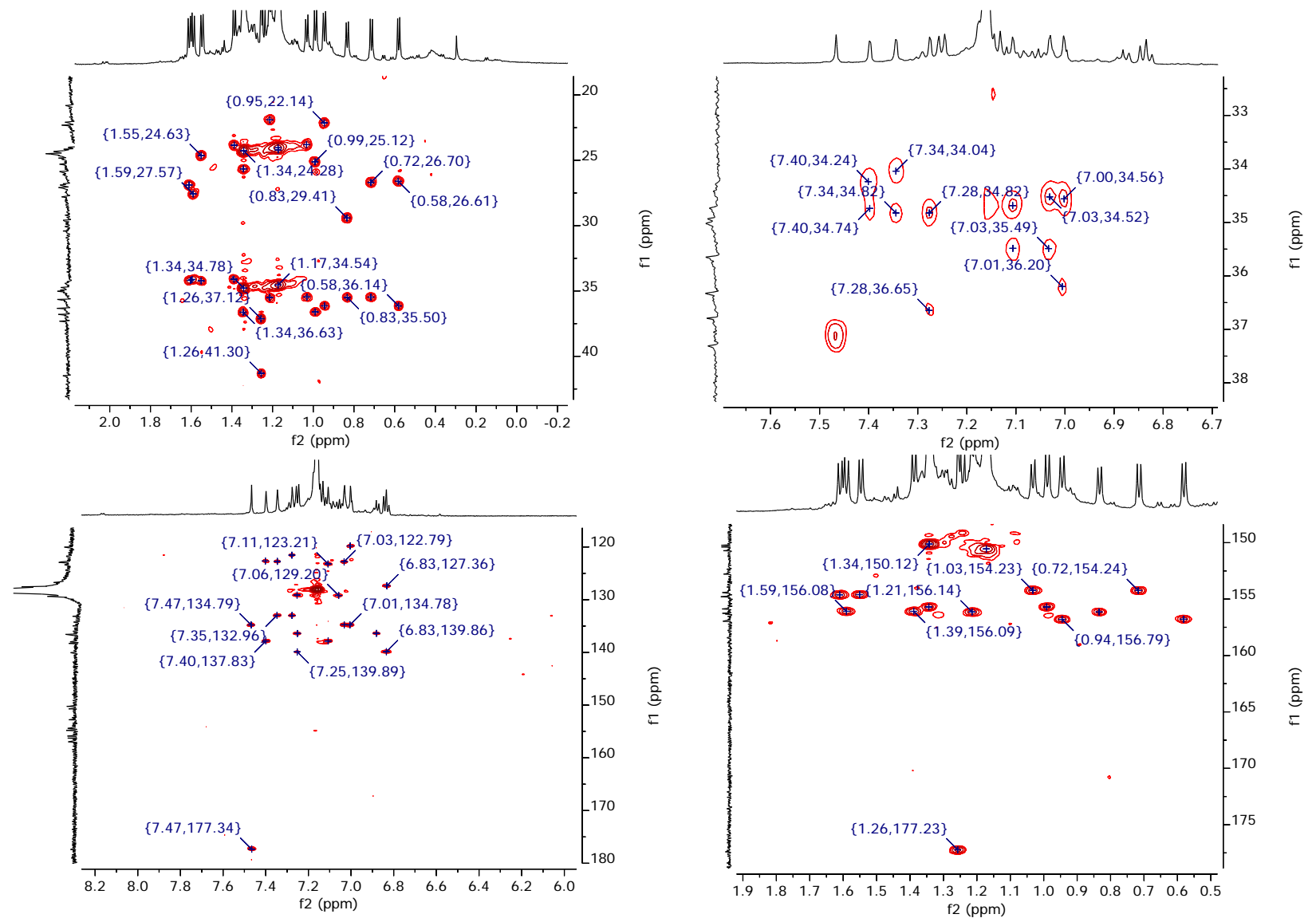
Figure S14: ${ }^{29} \mathrm{Si}-{ }^{1} \mathrm{H}$ gHMBC NMR spectrum of $9\left(600 \mathrm{MHz}, \mathrm{C}_{6} \mathrm{D}_{6}\right)$

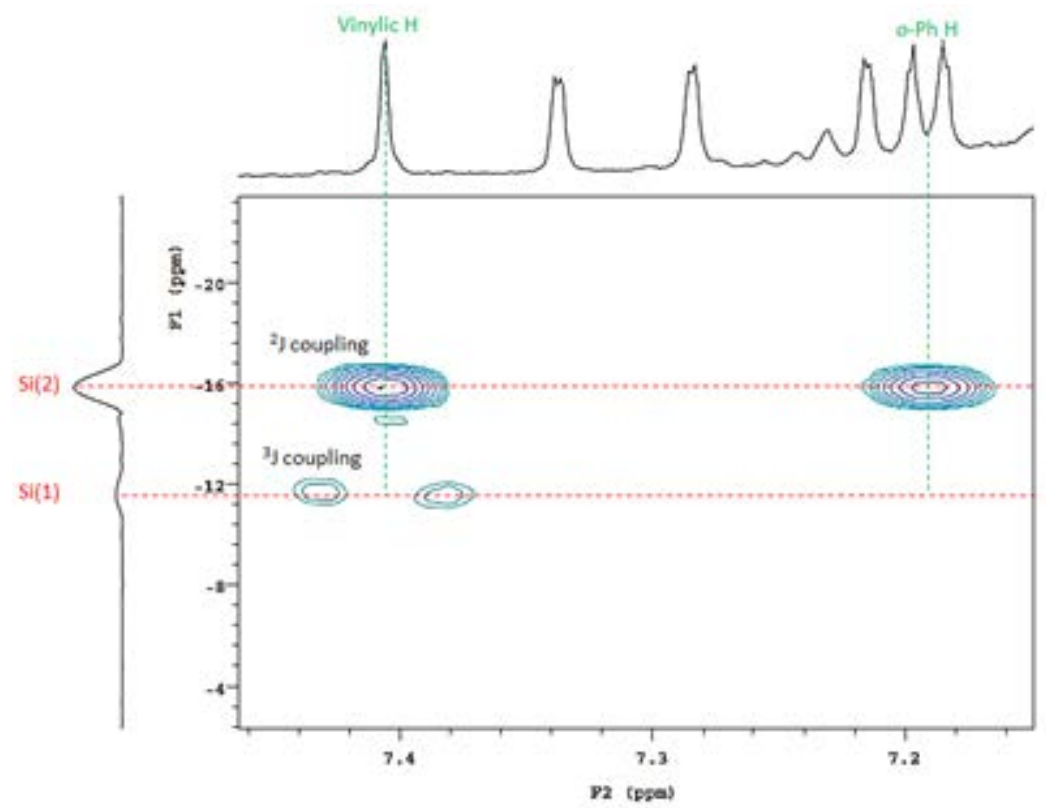




\section{Compound 10}

Yield $=6 \%{ }^{1} \mathrm{H} \mathrm{NMR}{ }^{6}\left(\mathrm{C}_{6} \mathrm{D}_{6}, 600 \mathrm{MHz}\right): \delta=7.47(\mathrm{~d}, J=7.6 \mathrm{~Hz}, 2 \mathrm{H}, o-\mathrm{Ph}-\mathrm{H}), 7.35(\mathrm{~d}, J=1.8$

Hz, 1 H, m-Tip-H), 7.32 (d, $J=1.8$ Hz, 1 H, m-Tip-H), 7.25 (d, J = 1.8 Hz, 1 H, m-Tip-H), 7.20 6.85 (m, Ph-H and 2x m-Tip-H), 7.09 (m-Tip-H), 7.00 (s, 1 H, HC=C), 3.95 and 3.94 (each sept, $J$ = 6.4 Hz, 2 H, iPr CH), 3.8 (CH-OMe), 3.75 (sept, $J=6.4$ Hz, iPr CH), 3.38 (sept, $J=6.4$ Hz, $i \operatorname{Pr}$ CH), 3.26 (s, 3 H, $\mathrm{OCH}_{3}$ ), 3.24 (m, iPr CH), 3.22 (m, iPr CH), 2.85 (sept, $J=6.4$ Hz, iPr CH), 2.80 - 2.70 (m, 2 x iPr CH), 2.66 (d, $J=5.8$ Hz, 1 H, CH-Ph), 1.59 (s, 3 H, CH $), 1.62,1.57$, 1.38, 1.32, 1.315, 1.29, 1.21, 1.20, 1.17, 1.16, 1.03, 1.01, 1.00, 0.98, 0.78, 0.72, 0.57 (all d, $J=6.7 \mathrm{~Hz}$, iPr $\left.\mathrm{CH}_{3}\right) .{ }^{13} \mathrm{C}$ NMR (151 MHz, $\left.\mathrm{C}_{6} \mathrm{D}_{6}\right) \delta 183.0(\mathrm{Si}-\mathrm{C}=\mathrm{CH}), 156.9,156.1,156.0,155.7,154.4,154.0$ (all Tip o-C), 150.4 (x2), 150.2 (all Tip p-C), 146.4 (Si-CH=C), 137.8, 132.3 (all Tip i-C), 129.9 (o-PhSi), 122.7 (Ph), 122.8, 122.4, 122.3, 121.3 (all Tip m-C), 71.7 (CHOMe) 58.2 (OMe), 38.2 (CHPh), 36.5, 35.9, 35.3 (x2), 34.5 (x3), 34.4, 33.8 (all iPr CH), 32.3 ( $\mathrm{CH}_{3} \mathrm{C}_{(\text {Cyclopropyl) }}$ 28.8, 27.3, 26.5, 26.3, 25.7 (x2), 24.8, 24.3, 24 (x5), 23.8, 21.8 (all $\left.i \mathrm{Pr} \mathrm{CH}_{3}\right), 13.6\left(\mathrm{CH}_{3(\text { Cyclopropyl)). }}{ }^{29} \mathrm{Si} \mathrm{NMR}\right.$ $\left(\mathrm{C}_{6} \mathrm{D}_{6}\right): \delta=-14\left(\mathrm{SiTip}_{2},{ }^{3} \mathrm{~J}_{\mathrm{Si}-\mathrm{H}}=28 \mathrm{~Hz}\right),-16$ (SiPhTip). ESI-MS m/z 951.6296 (calcd for $\left.\mathrm{C}_{64} \mathrm{H}_{88} \mathrm{NaOSi}_{2}, 951.6271\right)$.

\footnotetext{
${ }^{6}$ Integration values only shown for isolated signals. Overlapping signals are assigned by 2D NMR spectroscopy but not integrated.
} 
Figure S15: ${ }^{1} \mathrm{H}$ NMR Spectrum of $10\left(600 \mathrm{MHz}, \mathrm{C}_{6} \mathrm{D}_{6}\right)$

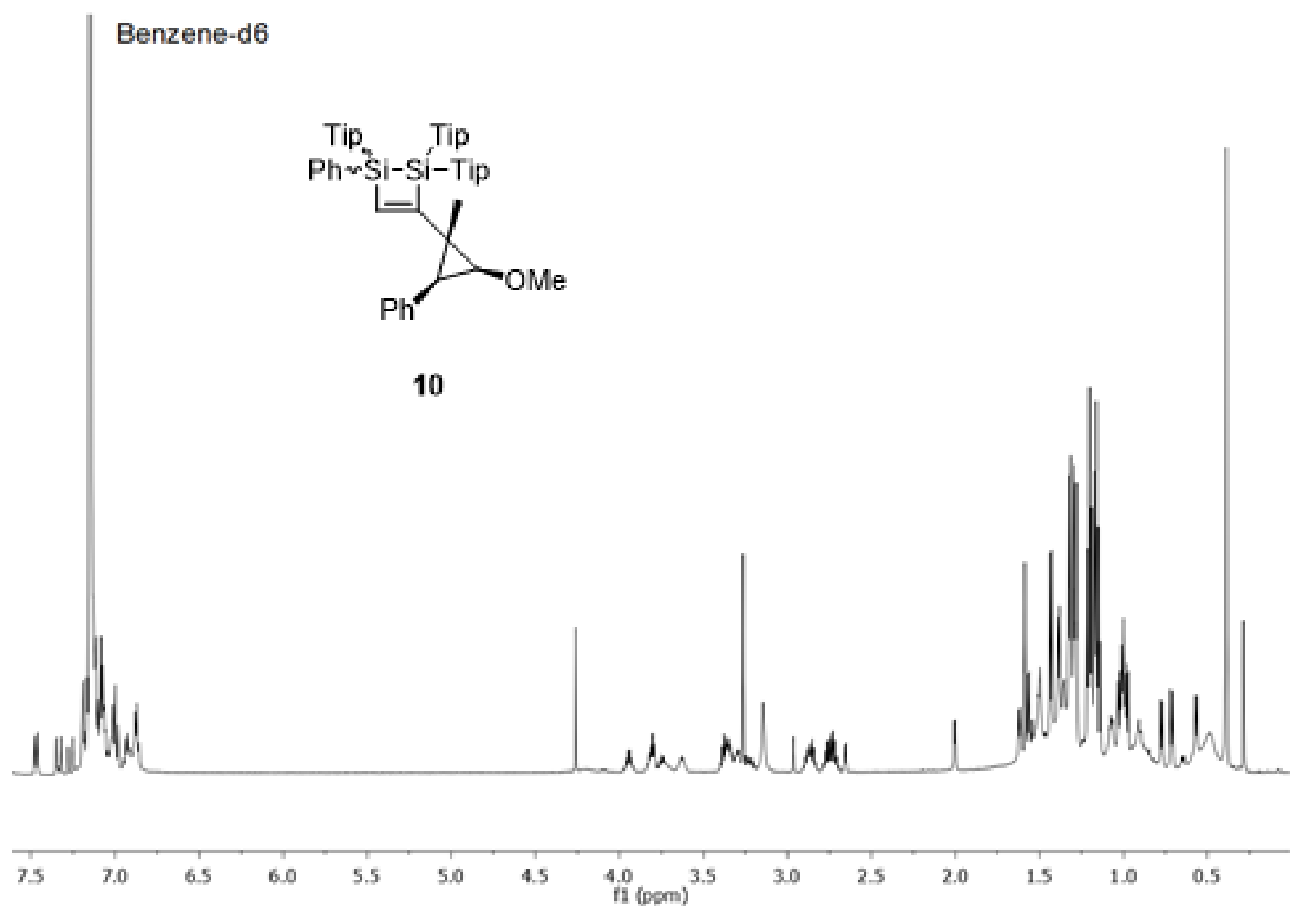


Figure S16: Expansions of the ${ }^{13} \mathrm{C}-{ }^{1} \mathrm{H}$ gHSQCAD NMR spectrum of $10\left(600 \mathrm{MHz}, \mathrm{C}_{6} \mathrm{D}_{6}\right)$ with ${ }^{1} \mathrm{H} ;{ }^{13} \mathrm{C}$ ranges of: $(0.5$ - 1.7 ppm; 14 -29 ppm) (2.6 - 4.0 ppm; 30 - 72 ppm) (6.8 - 7.6 ppm; 115 $-147 \mathrm{ppm})$

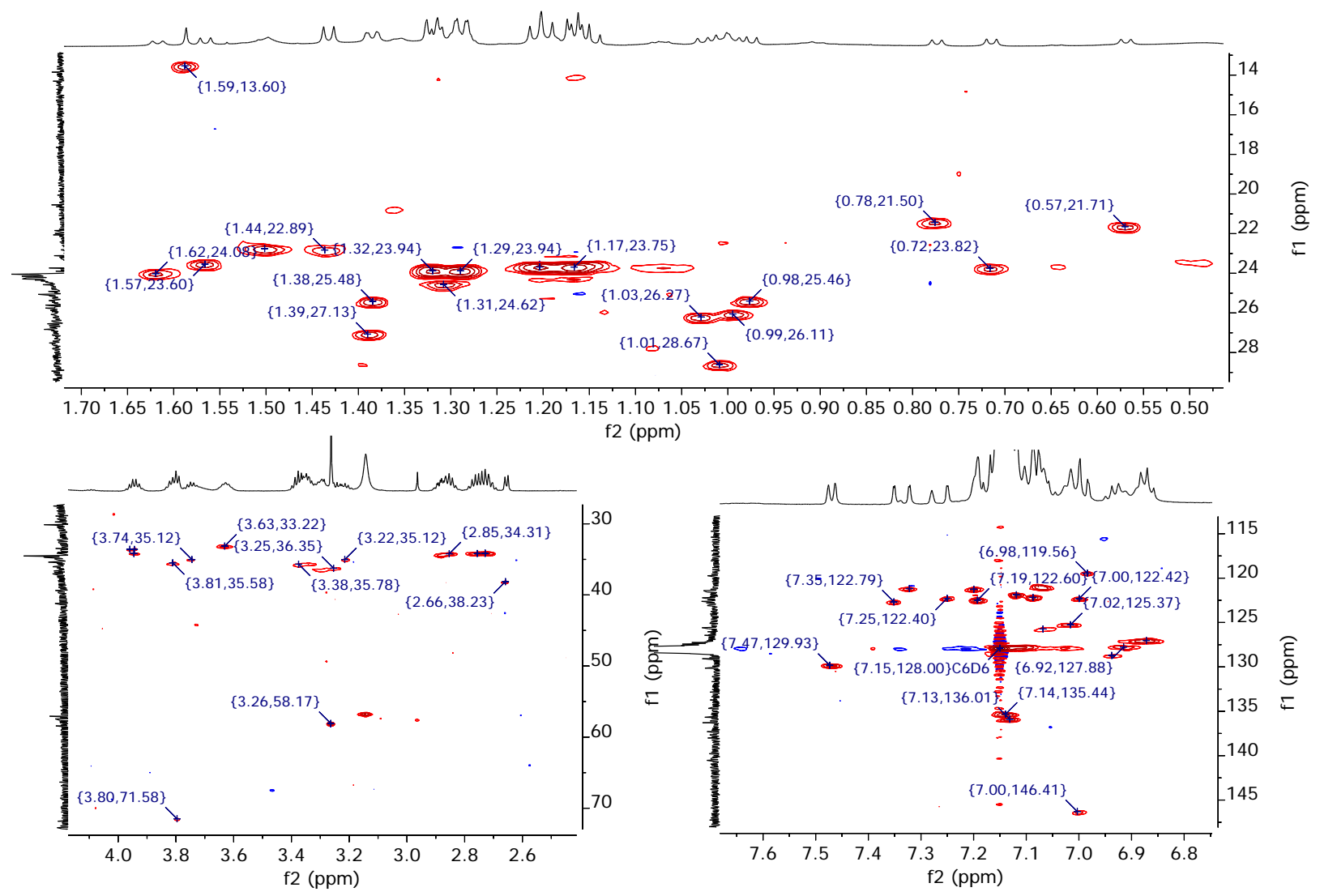


Figure S17: Expansions of the ${ }^{13} \mathrm{C}-{ }^{1} \mathrm{H}$ gHMBC NMR spectrum of 10 (600 $\mathrm{MHz}, \mathrm{C}_{6} \mathrm{D}_{6}$ ) with ${ }^{1} \mathrm{H} ;{ }^{13} \mathrm{C}$ ranges of: $(0.5$ - 1.8 ppm; 10 - 80 ppm) (6.8 - 7.5 ppm; 120 - 185 ppm) (0.3 - 1.7 ppm; $150-158 \mathrm{ppm})$

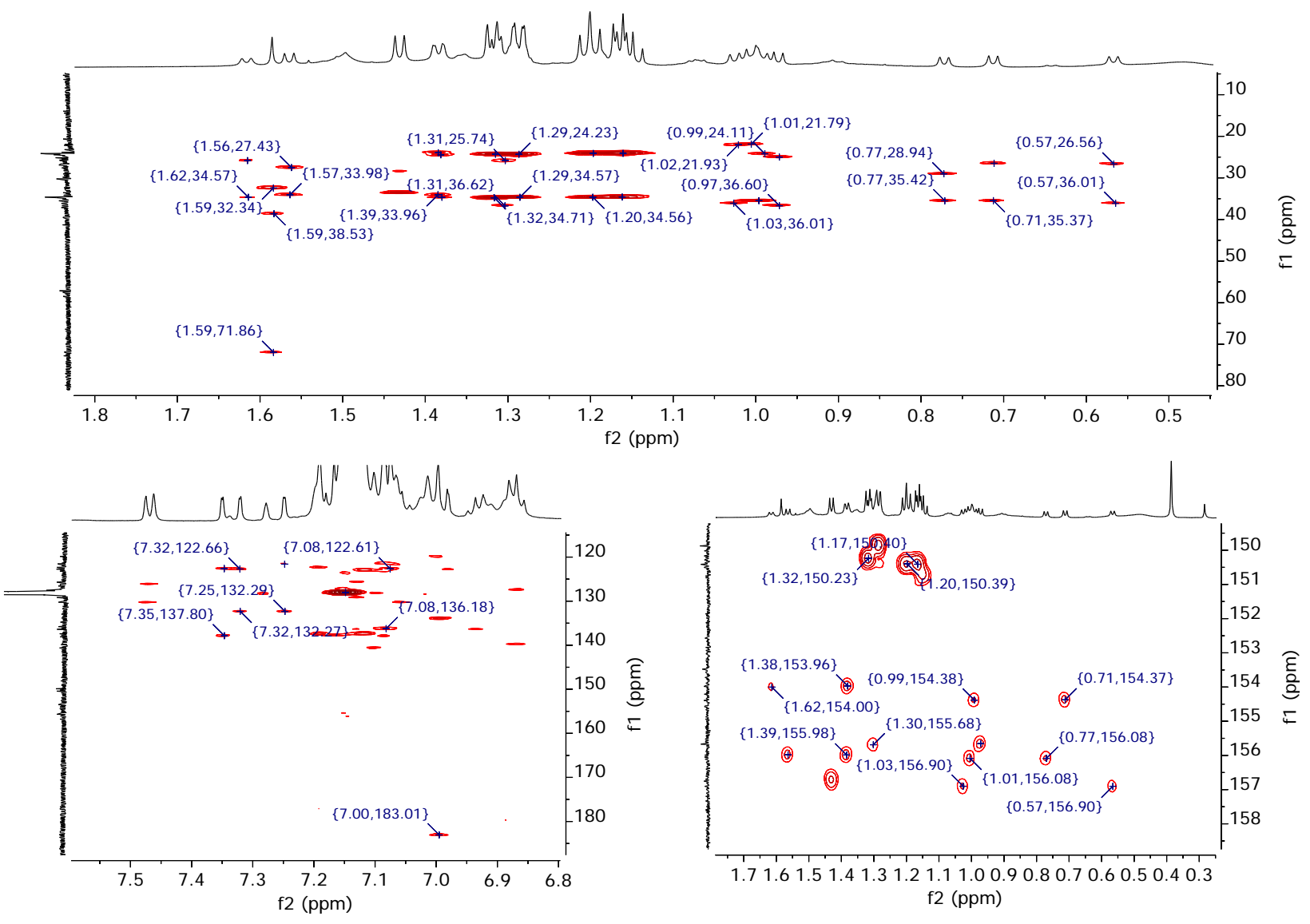


Figure S18: ${ }^{29} \mathrm{Si}-{ }^{1} \mathrm{H}$ gHMBC NMR spectrum of $\mathbf{1 0}\left(600 \mathrm{MHz}, \mathrm{C}_{6} \mathrm{D}_{6}\right)$

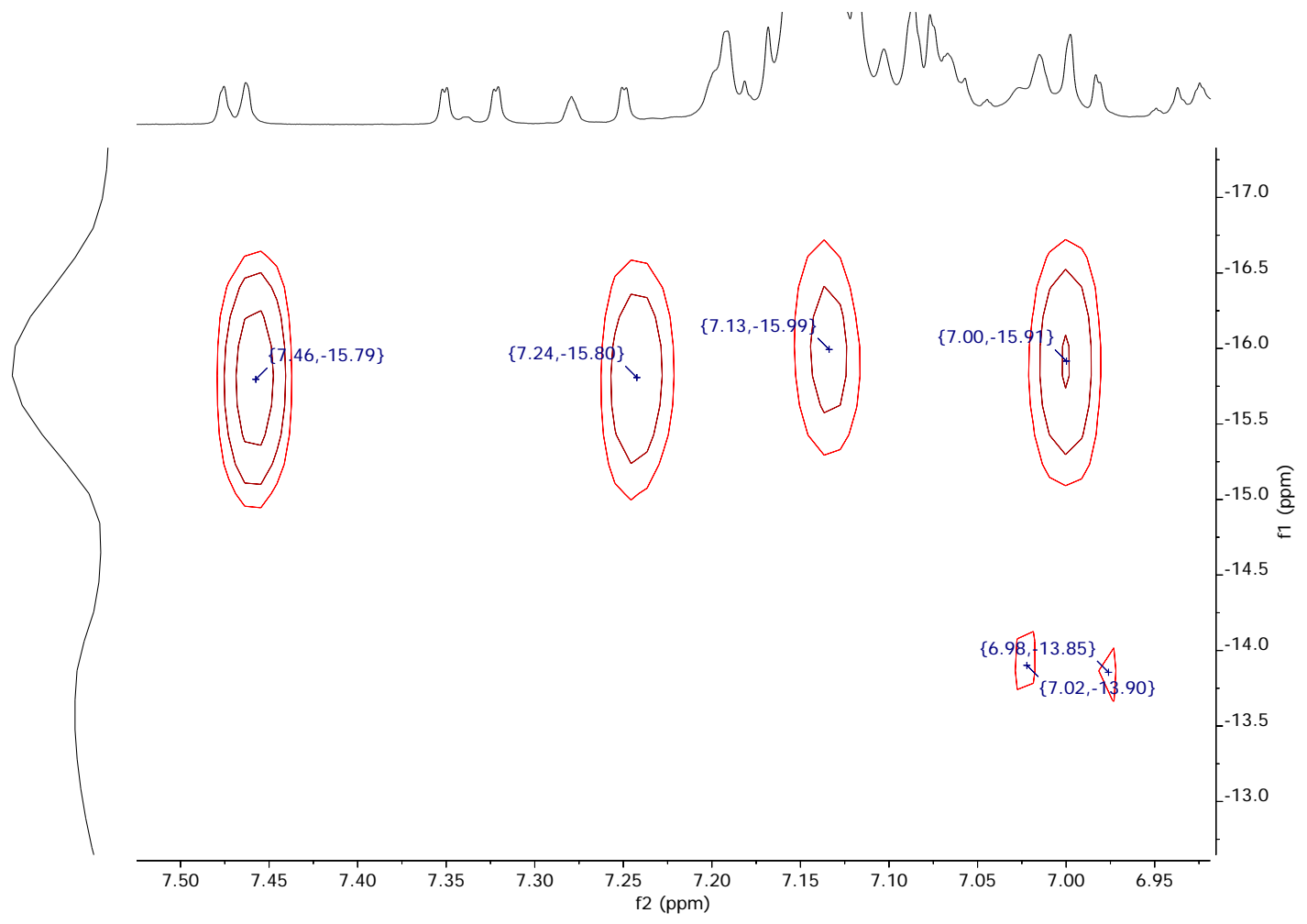




\section{X-Ray Crystallography}

Data Collection and Processing. The sample was mounted on a Mitegen polyimide micromount with a small amount of Paratone N oil. All X-ray measurements were made on a Nonius Bruker KappaCCD Apex2 diffractometer at a temperature of $110 \mathrm{~K}$. The unit cell dimensions were determined from a symmetry constrained fit of 9995 reflections with $7.12^{\circ}<2 \theta<128.02^{\circ}$. The data collection strategy was a number of $\omega$ and $\varphi$ scans which collected data up to $128.808^{\circ}(2 \theta)$. The frame integration was performed using SAINT. ${ }^{2}$ The resulting raw data were scaled and absorption corrected using a multi-scan averaging of symmetry equivalent data using SADABS. ${ }^{3}$ Structure Solution and Refinement. The structure was solved by using a dual space methodology using the SHELXT program. ${ }^{4}$ All non-hydrogen atoms were obtained from the initial solution. The hydrogen atoms were introduced at idealized positions and were allowed to ride on the parent atom. Several regions of disorder were found at the para isopropyl groups of the Tip ligands. Most involved an inversion at the methine carbon of the isopropyl groups and refined to various normalized occupancies (0.607(12) for C23; 0.545(11) for C87'; 0.65(3) for C11A). The structural model was fit to the data using full matrix least-squares based on $F^{2}$. The calculated structure factors included corrections for anomalous dispersion from the usual tabulation. The structure was refined using the SHELXL-2014 program from the SHELXTL suite of crystallographic software. ${ }^{5}$ Graphic plots were produced using the XP program suite. ${ }^{6}$ 
Table S3: Summary of crystallographic parameters for $\mathbf{7}$

\begin{tabular}{|c|c|}
\hline Formula & $\mathrm{C}_{64} \mathrm{H}_{88} \mathrm{OSi}_{2}$ \\
\hline Formula Weight $(\mathrm{g} / \mathrm{mol})$ & 929.52 \\
\hline Crystal Dimensions $(\mathrm{mm})$ & $0.309 \times 0.214 \times 0.134$ \\
\hline Crystal Colour and Habit & colourless prism \\
\hline Crystal System & triclinic \\
\hline Space Group & $\mathrm{P}-1$ \\
\hline Temperature, $\mathrm{K}$ & 110 \\
\hline$a, \AA$ & $11.657(2)$ \\
\hline$b, \AA$ & $22.035(4)$ \\
\hline$c, \AA$ & $24.824(4)$ \\
\hline$a,{ }^{\circ}$ & 64.488(9) \\
\hline$\beta,^{\circ}$ & $77.996(10)$ \\
\hline$\gamma,{ }^{\circ}$ & $81.660(7)$ \\
\hline $\mathrm{V}, \AA^{3}$ & $5617.9(18)$ \\
\hline Number of reflections to determine final unit cell & 9995 \\
\hline Min and Max $2 \theta$ for cell determination, ${ }^{\circ}$ & 7.12, 128.02 \\
\hline Z & 4 \\
\hline $\mathrm{F}(000)$ & 2032 \\
\hline$\rho(\mathrm{g} / \mathrm{cm})$ & 1.099 \\
\hline$\lambda, \AA,(\mathrm{CuKa})$ & 1.54178 \\
\hline$\mu,\left(\mathrm{cm}^{-1}\right)$ & 0.858 \\
\hline Diffractometer Type & Nonius Bruker KappaCCD Apex2 \\
\hline Scan Type(s) & phi and omega scans \\
\hline Max $2 \theta$ for data collection, ${ }^{\circ}$ & 128.808 \\
\hline Measured fraction of data & 0.985 \\
\hline Number of reflections measured & 69911 \\
\hline Unique reflections measured & 18577 \\
\hline $\mathrm{R}_{\text {merge }}$ & 0.0646 \\
\hline Number of reflections included in refinement & 18577 \\
\hline Cut off Threshold Expression & $\mathrm{I}>2 \sigma(\mathrm{I})$ \\
\hline
\end{tabular}




\begin{tabular}{|l|l|}
\hline Structure refined using & full matrix least-squares using $\mathrm{F}^{2}$ \\
\hline Weighting Scheme & $\begin{array}{l}\mathrm{W}=1 /\left[\sigma^{2}\left(\mathrm{Fo}^{2}\right)+(0.0622 \mathrm{P})^{2}+2.8121 \mathrm{P}\right] \\
\text { where } \mathrm{P}=\left(\mathrm{Fo}^{2}+2 \mathrm{Fc}^{2}\right) / 3\end{array}$ \\
\hline Number of parameters in least-squares & 1246 \\
\hline $\mathrm{R}_{1}$ & 0.0527 \\
\hline $\mathrm{wR}_{2}$ & 0.1295 \\
\hline $\mathrm{R}_{1}$ (all data) & 0.0724 \\
\hline $\mathrm{wR}_{2}$ (all data) & 0.1419 \\
\hline GOF & 1.048 \\
\hline Maximum shift/error & 0.001 \\
\hline Min \& Max peak heights on final $\Delta \mathrm{F}$ Map $\left(e^{-} / \AA\right)$ & $-0.392,0.447$ \\
\hline
\end{tabular}

Where:

$\mathrm{R}_{1}=\Sigma\left(\left|\mathrm{F}_{\mathrm{o}}\right|-\left|\mathrm{F}_{\mathrm{c}}\right|\right) / \Sigma \mathrm{F}_{\mathrm{o}}$

$\mathrm{wR}_{2}=\left[\Sigma\left(w\left(\mathrm{~F}_{\mathrm{o}}^{2}-\mathrm{F}_{\mathrm{c}}^{2}\right)^{2}\right) / \Sigma\left(w \mathrm{~F}_{\mathrm{o}}^{4}\right)\right]^{1 / 2}$

$\mathrm{GOF}=\left[\Sigma\left(w\left(\mathrm{~F}_{\mathrm{o}}{ }^{2}-\mathrm{F}_{\mathrm{c}}{ }^{2}\right)^{2}\right) /(\text { No. of reflns. - No. of params. })\right]^{1 / 2}$

\section{References}

1. Gottschling, S. E.; Milnes, K. K.; Jennings, M. C.; Baines, K. M.; Organometallics, 2005, 24, 3811-3814.

2. Bruker-AXS, SAINT version 2013.8, 2013, Bruker-AXS, Madison, WI 53711, USA

3. Bruker-AXS, SADABS version 2012.1, 2012, Bruker-AXS, Madison, WI 53711, USA

4. Sheldrick, G. M.; Acta Cryst. 2015, A71, 3-8

5. Sheldrick, G. M.; Acta Cryst. 2015, C71, 3-8

6. Bruker-AXS, XP version 2013.1, 2013, Bruker-AXS, Madison, WI 53711, USA 